\title{
The effects of time-dependent dissipation on the basins of attraction for the pendulum with oscillating support
}

\author{
James A. Wright ${ }^{1}$, Michele Bartuccelli ${ }^{1}$, Guido Gentile ${ }^{2}$ \\ ${ }^{1}$ Department of Mathematics, University of Surrey, Guildford, GU2 7XH, UK \\ ${ }^{2}$ Dipartimento di Matematica e Fisica, Università di Roma Tre, 00146 Roma, Italy
}

\begin{abstract}
We consider a pendulum with vertically oscillating support and time-dependent damping coefficient which varies until reaching a finite final value. Although it is the final value which determines which attractors eventually exist, however the sizes of the corresponding basins of attraction are found to depend strongly on the full evolution of the dissipation. In particular we investigate numerically how dissipation monotonically varying in time changes the sizes of the basins of attraction. It turns out that, in order to predict the behaviour of the system, it is essential to understand how the sizes of the basins of attraction for constant dissipation depend on the damping coefficient. For values of the parameters where the systems can be considered as a perturbation of the simple pendulum, which is integrable, we characterise analytically the conditions under which the attractors exist and study numerically how the sizes of their basins of attraction depend on the damping coefficient. Away from the perturbation regime, a numerical study of the attractors and the corresponding basins of attraction for different constant values of the damping coefficient produces a much more involved scenario: changing the magnitude of the dissipation causes some attractors to disappear either leaving no trace or producing new attractors by bifurcation, such as period doubling and saddle-node bifurcation. Finally we pass to the case of an initially non-constant damping coefficient, both increasing and decreasing to some finite final value, and we numerically observe the resulting effects on the sizes of the basins of attraction: when the damping coefficient varies slowly from a finite initial value to a different final value, without changing the set of attractors, the slower the variation the closer the sizes of the basins of attraction are to those they have for constant damping coefficient fixed at the initial value. Furthermore, if during the variation of the damping coefficient attractors appear or disappear, remarkable additional phenomena may occur. For instance it can happen that, in the limit of very large variation time, a fixed point asymptotically attracts the entire phase space, up to a zero measure set, even though no attractor with such a property exists for any value of the damping coefficient between the extreme values.
\end{abstract}

Keywords: action-angle variables, attractors, basins of attraction, dissipative systems, non-constant dissipation, periodic motions, simple pendulum.

Mathematical Subject Classification (2000) 34C60, 34C25, 37C60, 58F12, 70K40, 70K50.

\section{Introduction}

Consider the ordinary differential equations

$$
\ddot{x}+G(x, t)+\gamma \dot{x}=0, \quad \ddot{\theta}+F(\theta, t)+\gamma \dot{\theta}=0,
$$


where $(x, \dot{x}) \in \mathbb{R}^{2}$ and $(\theta, \dot{\theta}) \in \mathbb{T} \times \mathbb{R}$, with $\mathbb{T}=\mathbb{R} / 2 \pi \mathbb{Z}$. The functions $F$ and $G$ are smooth and $2 \pi$-periodic in time $t$ ( $F$ is also $2 \pi$-periodic in $\theta$ ); the dots denote derivatives with respect to time. Equations to describe the motion of one-dimensional physical systems are often of this form, in which case the functions $G(x, t)$ and $F(\theta, t)$ can be considered as an external driving force and the parameter $\gamma$ represents the damping coefficient, which we shall assume positive.

First, for convenience, let us summarise some of the already known ideas regarding systems of the form (1.1), which can be found in the literature [1, 3, 5. When $\gamma$ is fixed at zero, the system is Hamiltonian and no attractors are present. For $\gamma>0$ numerical experiments show that a finite set of attractors exist: this is consistent with Palis' conjecture [31, 16, 33. The number of attractors present and the percentage of phase space covered by their basins of attraction depend upon the chosen values of the parameters (perturbation parameter $\varepsilon$ and damping coefficient $\gamma$ ), but, for all values of the parameters, the union of the corresponding basins of attraction completely fill the phase space, up to a set of zero measure. Moreover if the system is a perturbation of an integrable system (perturbation regime), all attractors found numerically turn out to be either fixed points or periodic solutions with periods that are rational multiples of the forcing period (subharmonic solutions); we cannot exclude the presence of chaotic attractors [20, 15], but apparently they either do not arise or seem to be irrelevant.

Generally in the literature the damping coefficient is taken as constant, but in many physical systems it changes non-periodically over time. This can be due to several factors, such as the heating or cooling of a mechanical system and the wear out or rust on mechanical parts. Despite this, usually models and numerical simulations of such systems only take the final value of dissipation into account when calculating basins of attraction. The recent paper 3 puts forward the idea that, although the final value of dissipation determines which attractors exist, the relative sizes of their basins of attraction depend on the evolution of the dissipation. In particular the effect of dissipation increasing to some constant value over a given time span induces a significant change to the sizes of the basins of attraction in comparison to those when dissipation is constant.

Let us illustrate in more detail the phenomenology. Suppose that for two values $\gamma_{0}$ and $\gamma_{1}$ of the damping coefficient, with $\gamma_{0} \neq \gamma_{1}$, the same set of attractors exists. Provided the difference between the two values is sufficiently large, the relative sizes of the basins of attraction under the two coefficients will in general be appreciably different. If we allow the damping coefficient $\gamma$ to depend on time, $\gamma=\gamma(t)$, and vary from $\gamma_{0}$ to $\gamma_{1}$ over an initial period of time $T_{0}$, after which it remains constant at the value $\gamma_{1}$, then the sizes of the basins of attraction will be different from those where the system has constant coefficient $\gamma_{1}$ throughout. Moreover if $T_{0}$ is taken larger, the sizes of the basins of attraction tend towards those for the system under constant $\gamma=\gamma_{0}$ : this reflects the fact that the damping coefficient remains close to $\gamma_{0}$ for longer periods of time.

Now consider two values $\gamma_{0}$ and $\gamma_{1}$ of the damping coefficient for which the corresponding sets of attractors $\mathcal{A}_{0}$ and $\mathcal{A}_{1}$ are not the same. As a system evolving under dissipation is expected to have only finitely many attractors, there can only be a finite number of attractors which exist for one of the two values and not for the other one. What happens is that, by varying $\gamma(t)$ from $\gamma_{0}$ to $\gamma_{1}$, an attractor can either appear or disappear, and in the latter case it can disappear either without leaving any trace or being replaced by a new attractor by bifurcation. Suppose, for instance, that the only difference between $\mathcal{A}_{0}$ and $\mathcal{A}_{1}$ is that the attractor $a_{0} \in \mathcal{A}_{0}$ simply disappears, that is $\mathcal{A}_{0} \backslash \mathcal{A}_{1}=\left\{a_{0}\right\}$; then, if the time $T_{0}$ over which $\gamma(t)$ varies is large, each remaining attractor tends to have a basin of attraction not smaller than that it has for $\gamma$ fixed at $\gamma_{0}$ : the reason being, again, that the damping coefficient remains close to $\gamma_{0}$ for a long time and, moreover, the trajectories which would be attracted by $a_{0}$ at $\gamma=\gamma_{0}$ will move towards some other attractor when $a_{0}$ disappears. If, instead, the only difference between the sets of attractors $\mathcal{A}_{0}$ and $\mathcal{A}_{1}$ is that the attractor $a_{0} \in \mathcal{A}_{0}$ is replaced by an attractor $a_{1}$, say by period doubling bifurcation, then, letting $\gamma(t)$ vary from $\gamma_{0}$ to $\gamma_{1}$ over a sufficiently large time $T_{0}$ causes the size of the basin of attraction of $a_{1}$ to tend towards that of the basin of attraction that $a_{0}$ has for $\gamma=\gamma_{0}$.

We summarise our results by the following statements.

1. If $\mathcal{A}_{0}$, the set of attractors at $\gamma=\gamma_{0}$, is a subset of $\mathcal{A}_{1}$, the set of attractors which exist at $\gamma=\gamma_{1}$, that is $\mathcal{A}_{0} \subseteq \mathcal{A}_{1}$, then, as the time $T_{0}$ over which $\gamma(t)$ is varied from $\gamma_{0}$ to $\gamma_{1}$ is taken larger, the basins of attraction tend towards those when $\gamma$ is kept constant at $\gamma=\gamma_{0}$. In 
particular, if an attractor belongs to $\mathcal{A}_{1} \backslash \mathcal{A}_{0}$, then the larger $T_{0}$ the more negligible is the corresponding basin of attraction.

2. If the set of attractors at fixed $\gamma=\gamma_{1}$ is a proper subset of those which exist at $\gamma=\gamma_{0}$, that is $\mathcal{A}_{1} \subset \mathcal{A}_{0}$, then, as $T_{0}$ is taken larger, the basins of attraction for the attractors which exist at both $\gamma_{0}$ and $\gamma_{1}$ change so that for $\gamma(t)$ varying from $\gamma_{0}$ to $\gamma_{1}$ they tend to become greater than or equal to those for constant $\gamma=\gamma_{0}$.

3. If an attractor $a_{0}$ exists for $\gamma=\gamma_{0}$ but is destroyed as $\gamma(t)$ tends towards $\gamma_{1}$, and a new attractor $a_{1}$ is created from it by bifurcation (we will explicitly investigate the case of saddlenode or period doubling bifurcations), then the size of the basin of attraction of $a_{1}$, as $T_{0}$ is taken larger, tends towards that of $a_{0}$ at constant $\gamma=\gamma_{0}$.

4. If $\mathcal{A}_{01}$ is the set of attractors which exist at both $\gamma=\gamma_{0}$ and $\gamma=\gamma_{1}$, that is $\mathcal{A}_{01}=\mathcal{A}_{0} \cap \mathcal{A}_{1}$, and none of the elements in $\mathcal{A}_{0} \backslash \mathcal{A}_{01}$ are linked by bifurcation to elements in $\mathcal{A}_{1} \backslash \mathcal{A}_{01}$, then, as $T_{0}$ is taken larger, the phase space covered by the basins of attraction of the attractors which belong to $\mathcal{A}_{01}$ tends towards $100 \%$. Moreover, all such attractors have a basin of attraction larger than or equal to that they have when the coefficient of dissipation is fixed at $\gamma=\gamma_{0}$.

The main model used in [3] to convey some of the ideas above is a version of the forced cubic oscillator, which is of the form of the first equation in 1.1 , with $G(x, t)=(1+\varepsilon \cos t) x^{3}$. This system, considered in the perturbation regime (both $\varepsilon$ and $\gamma$ small), apart from the fixed point and as far as the numerics fortells, exhibits only oscillatory attractors with different periods depending on the parameter values. Also discussed in [3] is the relevance to the spin-orbit problem, describing an asymmetric ellipsoidal satellite moving in a Keplerian elliptic orbit around a planet [28]: the corresponding equations of motion are of the form of the second equation in (1.1), with the tidal friction term $\gamma(t)(\dot{\theta}-1)$ instead of $\gamma \dot{\theta}$, with $\gamma(t)$ slowly increasing in time because of the the cooling of the satellite.

In the present paper we wish to extend the discussion to the pendulum with periodically oscillating support [25, 32. The latter is a system which has been already extensively studied in the literature (we refer to [5] for a list of references): it offers a wide variety of dynamics and, because of the separatrix of the unperturbed system, in the perturbation regime, unlike the cubic oscillator, also includes rotatory attractors in addition to the oscillatory attractors. An important difference with respect to the results in 1 is the following. In 11, if an attractor exists for some value of $\gamma$, it is found to exist for smaller values of $\gamma$ too. This is not always true for the pendulum considered in the present paper, where we will see that, at least for some values of the parameters, both increasing and decreasing $\gamma$ can destroy attractors as well as create new ones. However, this occurs away from the perturbation regime, where the system can no longer be considered as a perturbation of an integrable one: the appearance and disappearance of attractors would occur also in the case of the cubic oscillator for larger values of the forcing. In addition to the case of increasing dissipation studied in [3], here we also include the case where the damping coefficient decreases to a constant value, which is appropriate for physical systems where joints are initially tight and require time to loosen. In this case similar phenomena are expected. For instance, as the value of variation time $T_{0}$ is taken larger, the amount of phase space covered by each of the basins of attraction should tend towards that corresponding to original value $\gamma_{0}$ of $\gamma$, providing the set of attractors remains the same.

The non-linear pendulum with vertically oscillating support is described by

$$
\ddot{\theta}+f(t) \sin \theta+\gamma \dot{\theta}=0, \quad f(t)=\left(\frac{g}{\ell}-\frac{b \omega^{2}}{\ell} f_{0}(\omega t)\right),
$$

where $f_{0}$ is a smooth $2 \pi$-periodic function and the parameters $\ell, b, \omega$ and $g$ represent the length, amplitude and frequency of the oscillations of the support and the gravitational acceleration, respectively, all of which remain constant; for the sake of simplicity we shall take $f_{0}(\omega t)=\cos (\omega t)$ in (1.2), as in [5, 6, 7]. As mentioned above, the parameter $\gamma$ represents the damping coefficient, 
which, for analysis where it remains constant, we shall model as $\gamma=C_{n} \varepsilon^{n}$, where $\varepsilon$ is small and $n$ is an integer. We shall consider $(1.2)$ as a pair of coupled first order non-autonomous differential equations by letting $x=\theta$ and $y=\dot{x}$, such that the phase space is $\mathbb{T} \times \mathbb{R}$ and the system can be written as

$$
\dot{x}=y, \quad \dot{y}=-\left(\frac{g}{\ell}-\frac{b \omega^{2}}{\ell} \cos (\omega t)\right) \sin x-\gamma y .
$$

The system described by $(1.2)$ can be non-dimensionalised by taking

$$
\alpha=\frac{g}{\ell \omega^{2}}, \quad \beta=\frac{b}{\ell}, \quad \tau=\omega t
$$

so that it becomes

$$
\theta^{\prime \prime}+f(\tau) \sin \theta+\gamma \theta^{\prime}=0, \quad f(\tau)=(\alpha-\beta \cos \tau),
$$

or, written as a system of first order differential equations,

$$
x^{\prime}=y, \quad y^{\prime}=-f(\tau) \sin x-\gamma y,
$$

where the dashes represent differentiation with respect to the new time $\tau$ and $\gamma$ has been normalised so as not to contain the frequency. Linearisation of the system about either fixed point results in a system of the form of Mathieu's equation, see for instance 27. When the downwards fixed point is linearly stable, it is possible, for certain parameter values, to prove analytically the conditions for which the fixed point attracts a full measure set of initial conditions; see Appendix A.

In the Sections that follow we shall use the non-dimensionalised version of the system $(1.3)$, preferable for numerical implementation as it reduces the number of parameters in the system. In Section 2 we detail the calculations of the threshold values for the attractors, that is the values of constant $\gamma$ below which periodic attractors exist in the perturbation regime (small $\beta$ ). As we shall see, because of the presence of the separatrix for the unperturbed pendulum, this will be of limited avail for practical purposes: the persisting periodic solutions found to first order are in general too close to the separatrix for the perturbation theory to converge. In Section 3 we present numerical results, in the case of both constant and non-constant (either increasing or decreasing) dissipation, for values of the parameters in the perturbation regime. Since for such values the downwards position turns out to be stable, we shall refer to this case as the downwards pendulum. Next, in Section 4 we perform the numerical analysis for values of the parameters for which the upwards position is stable (hence such a case will be referred to as the inverted pendulum). Of course such parameter values are far away from the perturbation regime: as a consequence additional phenomena occur, including period doubling and saddle-node bifurcations. In Section 5 we include a discussion of numerical methods used. Finally in Section 6 we draw our conclusions and briefly discuss some open problems and possible directions for future investigation.

\section{Thresholds values for the attractors}

The method used below to calculate the threshold values of $\gamma$ below which given attractors exist follows that described in [1, 3], where it was applied to the damped quartic oscillator and the spinorbit model. We consider the system (1.4), with $\beta=\varepsilon$ and $\gamma=C_{1} \varepsilon$, where $\varepsilon, C_{1}>0$. This approach is well suited to compute the leading order of the threshold values. In general, it would be preferable to write $\gamma$ as a function of $\varepsilon$ of the form $\gamma=C_{1} \varepsilon+C_{2} \varepsilon^{2}+\ldots$ (bifurcation curve), and fix the constants $C_{k}$ by imposing formal solubility of the equations to any perturbation order, see [18; however this only produces higher order corrections to the leading order value.

For $\varepsilon=0$ the system reduces to the simple pendulum $\theta^{\prime \prime}+\alpha \sin \theta=0$, which admits periodic solutions inside the separatrix (librations or oscillations) and outside the separatrix (rotations). In terms of the variables $(x, y)$ the equations $(1.4)$ become $x^{\prime}=y, y^{\prime}=-\sin x$ : the librations are described by

$$
\begin{cases}x_{\mathrm{osc}}(\tau)=2 \arcsin \left[k_{1} \operatorname{sn}\left(\sqrt{\alpha}\left(\tau-\tau_{0}\right), k_{1}\right)\right], & k_{1}<1, \\ y_{\mathrm{osc}}(\tau)=2 k_{1} \sqrt{\alpha} \operatorname{cn}\left(\sqrt{\alpha}\left(\tau-\tau_{0}\right), k_{1}\right), & \end{cases}
$$


while the rotations are described by

$$
\left\{\begin{array}{l}
x_{\mathrm{rot}}(\tau)=2 \arcsin \left[\operatorname{sn}\left(\sqrt{\alpha}\left(\tau-\tau_{0}\right) / k_{2}, k_{2}\right)\right], \\
y_{\mathrm{rot}}(\tau)=2 k_{2}^{-1} \sqrt{\alpha} \mathrm{dn}\left(\sqrt{\alpha}\left(\tau-\tau_{0}\right) / k_{2} \cdot k_{2}\right),
\end{array} \quad k_{2}<1,\right.
$$

where $\mathrm{cn}(\cdot, k), \operatorname{sn}(\cdot, k)$ and $\operatorname{dn}(\cdot, k)$ are the Jacobi elliptic functions with elliptic modulus $k$ [8, 11, 26, 35], and $k_{1}$ and $k_{2}$ are such that $k_{1}^{2}=(E+\alpha) / 2 \alpha$ and $k_{2}^{2}=1 / k_{1}^{2}$, with $E$ being the energy of the pendulum. From (2.1) and $(2.2)$ it can be seen that the solutions are functions of $\left(\tau-\tau_{0}\right)$, so that the phase of a solution depends on the initial conditions. We can fix the phase of the solution to zero without loss of generality by instead writing $f(\tau)$ in equation (1.4) as $f\left(\tau-\tau_{0}\right)$. This moves the freedom of choice in the initial condition to the phase of the forcing.

The dynamics of the simple pendulum can be conveniently written in terms of action-angle variables $(I, \varphi)$, for which we obtain two sets of variables: for the librations inside the separatrix one expresses the action as

$$
I=\frac{8}{\pi} \sqrt{\alpha}\left[\left(k_{1}^{2}-1\right) \mathbf{K}\left(k_{1}\right)+\mathbf{E}\left(k_{1}\right)\right]
$$

where $\mathbf{K}(k)$ and $\mathbf{E}(k)$ are the complete elliptic integrals of the first and second kind, respectively, and writes

$$
x=2 \arcsin \left[k_{1} \operatorname{sn}\left(\frac{2 \mathbf{K}\left(k_{1}\right)}{\pi} \varphi, k_{1}\right)\right], \quad y=2 k_{1} \sqrt{\alpha} \operatorname{cn}\left(\frac{2 \mathbf{K}\left(k_{1}\right)}{\pi} \varphi, k_{1}\right),
$$

with $k_{1}$ obtained by inverting $(2.3)$, while for the rotations outside the separatrix one expresses the actions as

$$
I=\frac{4}{k_{2} \pi} \sqrt{\alpha} \mathbf{E}\left(k_{2}\right),
$$

and writes

$$
x=2 \arcsin \left[\operatorname{sn}\left(\frac{\mathbf{K}\left(k_{2}\right)}{\pi} \varphi, k_{2}\right)\right], \quad y=\frac{2}{k_{2}} \sqrt{\alpha} \operatorname{dn}\left(\frac{\mathbf{K}\left(k_{2}\right)}{\pi} \varphi, k_{2}\right),
$$

with $k_{2}$ obtained by inverting (2.5); further details can be found in Appendix B.

For $\varepsilon$ small, in order to compute the thresholds values, we first write the equations of motion for the perturbed system in terms of the action-angle coordinates $(I, \varphi)$ of the simple pendulum, then we look for solutions in the form of power series expansions in $\varepsilon$,

$$
I(\tau)=\sum_{n=0}^{\infty} \varepsilon^{n} I^{(n)}(\tau), \quad \varphi(\tau)=\sum_{n=0}^{\infty} \varepsilon^{n} \varphi^{(n)}(\tau)
$$

where $I^{(0)}(\tau)$ and $\varphi^{(0)}(\tau)$ are the solutions to the unperturbed system, that is, see Appendix B $\left(I^{(0)}(\tau), \varphi^{(0)}(\tau)\right)=\left(I_{\mathrm{osc}}, \varphi_{\mathrm{osc}}(\tau)\right)$ and $\left(I^{(0)}(\tau), \varphi^{(0)}(\tau)\right)=\left(I_{\mathrm{rot}}, \varphi_{\mathrm{rot}}(\tau)\right)$, in the case of oscillations and rotations, respectively, with

$$
\begin{aligned}
& I_{\mathrm{osc}}=\frac{8}{\pi} \sqrt{\alpha}\left[\left(k_{1}^{2}-1\right) \mathbf{K}\left(k_{1}\right)+\mathbf{E}\left(k_{1}\right)\right], \quad \varphi_{\mathrm{osc}}(\tau)=\frac{\pi}{2 \mathbf{K}\left(k_{1}\right)} \sqrt{\alpha} \tau, \\
& I_{\mathrm{rot}}=\frac{4}{k_{2} \pi} \sqrt{\alpha} \mathbf{E}\left(k_{2}\right), \quad \varphi_{\mathrm{rot}}(\tau)=\frac{\pi}{\mathbf{K}\left(k_{2}\right)} \sqrt{\alpha} \frac{\tau}{k_{2}},
\end{aligned}
$$

and with given $k_{1}=k_{1}^{(0)}$ and $k_{2}=k_{2}^{(0)}$.

As the solution (2.7) is found using perturbation theory, its validity is restricted to the system where $\varepsilon$ is comparatively small. In particular this limitation has the result that the calculations of the threshold values are not valid for the inverted pendulum, where large $\varepsilon$ is required to stabilise the system. On the other hand the regime of small $\varepsilon$ has the advantage that we can characterise analytically the attractors and hence allows a better understanding of the dynamics with respect to the case of large $\varepsilon$, where only numerical results are available. 


\subsection{Librations}

We first write the equations of motion (1.4) in action-angle variables, see Appendix D as

$$
\begin{gathered}
\varphi^{\prime}=\frac{\pi \sqrt{\alpha}}{2 \mathbf{K}\left(k_{1}\right)}-\frac{\varepsilon \pi}{2 \mathbf{K}\left(k_{1}\right) \sqrt{\alpha}}\left[\operatorname{sn}^{2}(\cdot)+\frac{k_{1}^{2} \operatorname{sn}^{2}(\cdot) \mathrm{cn}^{2}(\cdot)}{1-k_{1}^{2}}-\frac{\mathbf{Z}(\cdot) \operatorname{sn}(\cdot) \operatorname{cn}(\cdot) \operatorname{dn}(\cdot)}{1-k_{1}^{2}}\right] \cos \left(\tau-\tau_{0}\right) \\
+\frac{C_{1} \varepsilon \pi \operatorname{cn}(\cdot)}{2 \mathbf{K}\left(k_{1}\right)}\left[\frac{\operatorname{sn}(\cdot)}{\operatorname{dn}(\cdot)}+\frac{k_{1}^{2} \operatorname{sn}(\cdot) \mathrm{cn}^{2}(\cdot)}{\left(1-k_{1}^{2}\right) \operatorname{dn}(\cdot)}-\frac{\mathbf{Z}(\cdot) \operatorname{cn}(\cdot)}{1-k_{1}^{2}}\right], \\
I^{\prime}=\frac{8 \varepsilon k_{1}^{2} \mathbf{K}\left(k_{1}\right)}{\pi} \cos \left(\tau-\tau_{0}\right) \operatorname{sn}(\cdot) \operatorname{cn}(\cdot) \operatorname{dn}(\cdot)-\frac{8 C_{1} \varepsilon k_{1}^{2} \sqrt{\alpha} \mathbf{K}\left(k_{1}\right)}{\pi} \operatorname{cn}^{2}(\cdot),
\end{gathered}
$$

where $\mathbf{Z}(\cdot)$ is the Jacobi zeta function, see [26]. Here and throughout Section 2.1 to save clutter we define $(\cdot)=\left(\frac{2 \mathbf{K}\left(k_{1}\right)}{\pi} \varphi, k_{1}\right)$. Note that in 2.9 , the dependence on $I$ of the vector field is through the variable $k_{1}$, according to 2.3 .

The coordinates for the unperturbed system $(\varepsilon=0)$ satisfy

$$
\varphi^{\prime}=\frac{\mathrm{d} E}{\mathrm{~d} I}:=\Omega(I)=\frac{\pi \sqrt{\alpha}}{2 \mathbf{K}\left(k_{1}\right)}, \quad I^{\prime}=0 .
$$

Linearising around $\left(\varphi^{(0)}(\tau), I^{(0)}(\tau)\right)=\left(\Omega\left(I^{(0)}\right) \tau, I^{(0)}\right)$, we have

$$
\delta \varphi^{\prime}=\frac{\partial \Omega}{\partial I}\left(I^{(0)}\right) \delta I, \quad \delta I^{\prime}=0
$$

where, see Appendix B.

$$
\zeta(I):=\frac{\partial \Omega}{\partial I}(I)=-\frac{\pi^{2}}{16 k_{1}^{2} \mathbf{K}^{3}\left(k_{1}\right)}\left[\frac{\mathbf{E}\left(k_{1}\right)}{1-k_{1}^{2}}-\mathbf{K}\left(k_{1}\right)\right] .
$$

Since $I=I\left(k_{1}\right)$, that is the action is a function of $k_{1}$, setting $I=I^{(0)}$ fixes $k_{1}=k_{1}^{(0)}$, yielding $\zeta\left(I^{(0)}\right)=\zeta^{(0)}$, with $\zeta^{(0)}$ given by (2.12) with $k_{1}=k_{1}^{(0)}$.

The linearised system 2.11) can by written in compact form as

$$
\left(\begin{array}{c}
\delta \varphi^{\prime} \\
\delta I^{\prime}
\end{array}\right)=\left(\begin{array}{cc}
0 & \zeta^{(0)} \\
0 & 0
\end{array}\right)\left(\begin{array}{c}
\delta \varphi \\
\delta I
\end{array}\right)
$$

The Wronskian matrix $W(\tau)$ is defined as the solution of the unperturbed linear system

$$
W^{\prime}(\tau)=\left(\begin{array}{cc}
0 & \zeta^{(0)} \\
0 & 0
\end{array}\right) W(\tau), \quad W(0)=\mathbb{I},
$$

where $\mathbb{I}$ is the $2 \times 2$ identity matrix. Hence

$$
W(\tau)=\left(\begin{array}{cc}
1 & \zeta^{(0)} \tau \\
0 & 1
\end{array}\right)
$$

with $(1,0)$ and $\left(\zeta^{(0)} \tau, 1\right)$ two linearly independent solutions to $(2.13)$.

We now look for periodic solutions $(\varphi(\tau), I(\tau))$ to 2.9 with period $T=2 \pi q=4 \mathbf{K}\left(k_{1}\right) p / \sqrt{\alpha}$, with $p / q \in \mathbb{Q}$, of the form (2.7); see also [18, 19] for a more general discussion. A solution of this kind will be referred to as a $p: q$ resonance.

The functions $\left(\varphi^{(n)}(\tau), I^{(n)}(\tau)\right)$ are formally obtained by introducing the expansions 2.7) into the equations (2.9) and equating the coefficients of order $n$. This leads to the equations

$$
\left(\begin{array}{c}
\left(\varphi^{(n)}\right)^{\prime} \\
\left(I^{(n)}\right)^{\prime}
\end{array}\right)=\left(\begin{array}{c}
\zeta^{(0)} I^{(n)} \\
0
\end{array}\right)+\left(\begin{array}{c}
F_{1}^{(n)}(\tau) \\
F_{2}^{(n)}(\tau)
\end{array}\right)
$$


with $F_{1}^{(n)}(\tau)$ and $F_{2}^{(n)}(\tau)$ given by

$$
\begin{gathered}
F_{1}^{(n)}(\tau)=\left[\frac{\pi \sqrt{\alpha}}{2 \mathbf{K}\left(k_{1}\right)}-\zeta^{(0)} I\right]^{(n)}+\left[-\frac{\pi}{2 \mathbf{K}\left(k_{1}\right) \sqrt{\alpha}}\left[\operatorname{sn}^{2}(\cdot)+\frac{k_{1}^{2}}{1-k_{1}^{2}} \operatorname{sn}^{2}(\cdot) \operatorname{cn}^{2}(\cdot)\right.\right. \\
\left.-\frac{\mathbf{Z}(\cdot)}{1-k_{1}^{2}} \operatorname{sn}(\cdot) \operatorname{cn}(\cdot) \operatorname{dn}(\cdot)\right] \cos \left(\tau-\tau_{0}\right) \\
\left.+\frac{C_{1} \pi \operatorname{cn}(\cdot)}{2 \mathbf{K}\left(k_{1}\right)}\left[\frac{\operatorname{sn}(\cdot)}{\operatorname{dn}(\cdot)}+\frac{k_{1}^{2} \operatorname{sn}(\cdot) \operatorname{cn}^{2}(\cdot)}{\left(1-k_{1}^{2}\right) \operatorname{dn}(\cdot)}-\frac{\mathbf{Z}(\cdot) \operatorname{cn}(\cdot)}{1-k_{1}^{2}}\right]\right]^{(n-1)}, \\
F_{2}^{(n)}(\tau)=\left[\frac{8 k_{1}^{2} \mathbf{K}\left(k_{1}\right)}{\pi} \cos \left(\tau-\tau_{0}\right) \operatorname{sn}(\cdot) \operatorname{cn}(\cdot) \operatorname{dn}(\cdot)-\frac{8 C_{1} k_{1}^{2} \sqrt{\alpha} \mathbf{K}\left(k_{1}\right)}{\pi} \operatorname{cn}^{2}(\cdot)\right]^{(n-1)} .
\end{gathered}
$$

The notation $[\ldots]^{(n)}$ means that one has to take all terms of order $n$ in $\varepsilon$ of the function inside [...]. By construction, $F_{1}^{(n)}(\tau)$ and $F_{2}^{(n)}(\tau)$ depend only on the coefficients $\varphi^{(p)}(\tau)$ and $I^{(p)}(\tau)$, with $p<n$, so that 2.15) can be solved recursively.

Then, by using the Wronskian matrix 2.14, see [30, one can integrate 2.15) so as to obtain

$$
\left(\begin{array}{c}
\varphi^{(n)}(\tau) \\
I^{(n)}(\tau)
\end{array}\right)=W(\tau)\left(\begin{array}{c}
\bar{\varphi}^{(n)} \\
\bar{I}^{(n)}
\end{array}\right)+W(\tau) \int_{0}^{\tau} \mathrm{d} \sigma W^{-1}(\sigma)\left(\begin{array}{c}
F_{1}^{(n)}(\sigma) \\
F_{2}^{(n)}(\sigma)
\end{array}\right)
$$

where $\bar{\varphi}^{(n)}$ and $\bar{I}^{(n)}$ are the $n^{\text {th }}$ order in the $\varepsilon$-expansion of the initial conditions for $\varphi$ and $I$, respectively. In the last term of 2.16 we have

$$
W(\tau) \int_{0}^{\tau} \mathrm{d} \sigma W^{-1}(\sigma)\left(\begin{array}{l}
F_{1}^{(n)}(\sigma) \\
F_{2}^{(n)}(\sigma)
\end{array}\right)=\int_{0}^{\tau} \mathrm{d} \sigma W(\tau-\sigma)\left(\begin{array}{l}
F_{1}^{(n)}(\sigma) \\
F_{2}^{(n)}(\sigma)
\end{array}\right) .
$$

This yields

$$
\begin{aligned}
& \varphi^{(n)}(\tau)=\bar{\varphi}^{(n)}+\zeta^{(0)} \tau \bar{I}^{(n)}+\int_{0}^{\tau} \mathrm{d} \sigma F_{1}^{(n)}(\sigma)+\zeta^{(0)} \int_{0}^{\tau} \mathrm{d} \sigma \int_{0}^{\sigma} \mathrm{d} \sigma^{\prime} F_{2}^{(n)}\left(\sigma^{\prime}\right), \\
& I^{(n)}(\tau)=\bar{I}^{(n)}(\tau)+\int_{0}^{\tau} \mathrm{d} \sigma F_{2}^{(n)}(\sigma) .
\end{aligned}
$$

For a periodic function $g$, let us denote the average of $g$ with $\langle g\rangle$ and the zero-average function $g-\langle g\rangle$ with $\breve{g}$. Suppose that

$$
\left\langle F_{2}^{(n)}\right\rangle:=\frac{1}{T} \int_{0}^{T} \mathrm{~d} \tau F_{2}^{(n)}(\tau)=0,
$$

where $T=4 \mathbf{K}\left(k_{1}\right) p$; we will check later on the validity of 2.18 . Then we may write

$$
\begin{aligned}
& \mathscr{F}_{1}^{(n)}(\tau)=\int_{0}^{\tau} \mathrm{d} \sigma F_{1}^{(n)}(\sigma)=\left\langle F_{1}^{(n)}\right\rangle \tau+\int_{0}^{\tau} \mathrm{d} \sigma \breve{F}_{1}^{(n)}(\sigma), \\
& \mathscr{F}_{2}^{(n)}(\tau)=\int_{0}^{\tau} F_{2}^{(n)}(\sigma) \mathrm{d} \sigma=\int_{0}^{\tau} \mathrm{d} \sigma \breve{F}_{2}^{(n)}(\sigma),
\end{aligned}
$$

and subsequently rewrite 2.17) as

$$
\begin{aligned}
& \varphi^{(n)}(\tau)=\bar{\varphi}^{(n)}+\zeta^{(0)} \tau \bar{I}^{(n)}+\left\langle F_{1}^{(n)}\right\rangle \tau+\int_{0}^{\tau} \mathrm{d} \sigma \breve{F}_{1}^{(n)}(\sigma)+\zeta^{(0)}\left\langle\mathscr{F}_{2}^{(n)}\right\rangle \tau+\zeta^{(0)} \int_{0}^{\tau} \mathrm{d} \sigma \mathscr{F}_{2}^{(n)}(\sigma), \\
& I^{(n)}(\tau)=\bar{I}^{(n)}+\int_{0}^{\tau} \mathrm{d} \sigma \breve{F}_{2}^{(n)}(\sigma),
\end{aligned}
$$

in which all the terms which are not linear in $\tau$ are periodic. If we choose our initial conditions $\bar{I}^{(n)}$ such that they satisfy

$$
\bar{I}^{(n)}=-\frac{1}{\zeta^{(0)}}\left\langle F_{1}^{(n)}\right\rangle-\left\langle\mathscr{F}_{2}^{(n)}\right\rangle,
$$


the above reduces to

$$
\begin{aligned}
& \varphi^{(n)}(\tau)=\bar{\varphi}^{(n)}(\tau)+\int_{0}^{\tau} \mathrm{d} \sigma \breve{F}_{1}^{(n)}(\sigma)+\zeta^{(0)} \int_{0}^{\tau} \mathrm{d} \sigma \mathscr{F}_{2}^{(n)}(\sigma), \\
& I^{(n)}(\tau)=\bar{I}^{(n)}+\int_{0}^{\tau} \mathrm{d} \sigma \breve{F}_{2}^{(n)}(\tilde{\tau}),
\end{aligned}
$$

so that both $\varphi^{(n)}(\tau)$ and $I^{(n)}(\tau)$ are periodic functions with period $T$, provided 2.18 holds.

Lemma 1 Consider the series (2.7). If $p / q=1 / 2 m, m \in \mathbb{N}$ and $C_{1}$ is small enough, then it is possible to fix the initial conditions $\left(\bar{\varphi}^{(n)}, \bar{I}^{(n)}\right)$ in such a way that 2.18 holds for all $n \geq 1$. If $p / q \neq 1 / 2 m$ for all $m \in \mathbb{N}$, then $(2.18)$ can be satisfied only for $C_{1}=0$.

Proof For $n=1$ we have

$$
\begin{aligned}
F_{2}^{(1)}(\tau) & =\frac{8 k_{1}^{2} \mathbf{K}\left(k_{1}\right)}{\pi} \cos \left(\tau-\tau_{0}\right) \operatorname{sn}\left(\sqrt{\alpha} \tau, k_{1}\right) \operatorname{cn}\left(\sqrt{\alpha} \tau, k_{1}\right) \operatorname{dn}\left(\sqrt{\alpha} \tau, k_{1}\right) \\
& -\frac{8 C_{1} k_{1}^{2} \sqrt{\alpha} \mathbf{K}\left(k_{1}\right)}{\pi} \operatorname{cn}^{2}\left(\sqrt{\alpha} \tau, k_{1}\right),
\end{aligned}
$$

with $k_{1}=k_{1}^{(0)}$ here and henceforth. Moreover set, see Appendix E.

$$
\begin{aligned}
\Delta & :=\frac{\sqrt{\alpha}}{4 \mathbf{K}\left(k_{1}\right)} \int_{0}^{4 \mathbf{K}\left(k_{1}\right) / \sqrt{\alpha}} \mathrm{d} \tau \operatorname{cn}^{2}\left(\sqrt{\alpha} \tau, k_{1}\right) \\
& =\frac{1}{2 \mathbf{K}\left(k_{1}\right)} \int_{0}^{2 \mathbf{K}\left(k_{1}\right)} \mathrm{d} \tau \operatorname{cn}^{2}\left(\tau, k_{1}\right)=\frac{1}{k_{1}^{2}}\left[\frac{1}{2 \mathbf{K}\left(k_{1}\right)} \mathbf{E}\left(2 \mathbf{K}\left(k_{1}\right), k_{1}\right)-\left(1-k_{1}^{2}\right)\right],
\end{aligned}
$$

where $\mathbf{E}(u, k)$ is the incomplete elliptic integral of the second kind, and $\Gamma_{1}\left(\tau_{0} ; p, q\right):=\sin \left(\tau_{0}\right) G_{1}(p, q)$, with

$$
\begin{aligned}
G_{1}(p, q) & =\frac{1}{T} \int_{0}^{T} \operatorname{sn}\left(\sqrt{\alpha} \tau, k_{1}\right) \operatorname{cn}\left(\sqrt{\alpha} \tau, k_{1}\right) \operatorname{dn}\left(\sqrt{\alpha} \tau, k_{1}\right) \sin (\tau) \\
& =\frac{1}{4 \mathbf{K}\left(k_{1}\right) p} \int_{0}^{4 \mathbf{K}\left(k_{1}\right) p} \mathrm{~d} \tau \operatorname{sn}\left(\tau, k_{1}\right) \operatorname{cn}\left(\tau, k_{1}\right) \operatorname{dn}\left(\tau, k_{1}\right) \sin (\tau / \sqrt{\alpha})
\end{aligned}
$$

Under the resonance condition $\pi \alpha / 2 \mathbf{K}\left(k_{1}\right)=p / q$, one has

$$
\sin (\tau / \sqrt{\alpha})=\sin \left(\frac{\pi \tau}{2 \mathbf{K}\left(k_{1}\right)} \frac{q}{p}\right),
$$

where $p$ and $q$ are relatively prime. By expanding the Jacobi elliptic functions in Fourier series, see Appendix E we find that $p, q$ must also satisfy the condition

$$
p\left( \pm\left(2 m_{1}-1\right) \pm\left(2 m_{2}-1\right) \pm 2 m_{3}\right) \pm q=0
$$

for $G_{1}(p, q)$ to be non-zero. Thus $q=2 m p, m \in \mathbb{N}$, that is $p=1$ and $q \in 2 \mathbb{N}$, and $\left\langle F_{2}^{(1)}\right\rangle=0$ provided $C_{1}$ and $\tau_{0}$ satisfy

$$
C_{1}=\frac{\sin \left(\tau_{0}\right)}{\sqrt{\alpha} \Delta} G_{1}(p, q)
$$

Note that the existence of a value of $\tau_{0}$ satisfying 2.21 is possible only if

$$
\left|C_{1}\right| \leq C_{1}(p / q):=\frac{1}{\sqrt{\alpha} \Delta} G_{1}(p, q) .
$$

Some values of the constants $C_{1}(p / q)$ for $\alpha=0.5$ are listed in Table 1 


\begin{tabular}{|ccccc|}
\hline$q$ & $k_{1}$ & $G_{1}(1 / q)$ & $\Delta$ & $C_{1}(1 / q)$ \\
\hline 2 & 0.8852015688846 & 0.172135 & 0.407121 & 0.597944 \\
4 & 0.998888384493 & 0.077675 & 0.224342 & 0.489649 \\
6 & 0.999986981343 & 0.051734 & 0.150043 & 0.487616 \\
8 & 0.999999846887 & 0.038800 & 0.112539 & 0.487578 \\
10 & 0.999999998199 & 0.031040 & 0.090032 & 0.487577 \\
12 & 0.999999999979 & 0.025867 & 0.075026 & 0.487577 \\
\hline
\end{tabular}

Table 1: Constants for the oscillating attractors with $\alpha=0.5$.

For all $n \geq 2$ we can write $F_{2}^{(n)}(\tau)$ as

$$
F_{2}^{(n)}(\tau)=\left.\frac{8 k_{1}^{2} \mathbf{K}\left(k_{1}\right)}{\pi} \frac{\partial}{\partial \varphi}\left(\cos \left(\tau-\tau_{0}\right) \operatorname{sn}(\cdot) \operatorname{cn}(\cdot) \operatorname{dn}(\cdot)-\sqrt{\alpha} C_{1} \operatorname{cn}^{2}(\cdot)\right)\right|_{\varphi=\varphi(0)} \bar{\varphi}^{(n-1)}+R^{(n)}(\tau),
$$

where $R^{(n)}(\tau)$ is a suitable function which does not depend on $\bar{\varphi}^{(n-1)}$. It can be seen that $\left\langle F_{2}^{(n)}\right\rangle=0$ if and only if

$$
\begin{gathered}
\left\langle R^{(n)}\right\rangle=-\frac{8 k_{1}^{2} \mathbf{K}\left(k_{1}\right)}{\pi}\left(\frac{1}{T} \int_{0}^{T} \mathrm{~d} \tau \frac{2 \mathbf{K}\left(k_{1}\right)}{\sqrt{\alpha} \pi} \frac{\partial}{\partial \tau}(\operatorname{sn}(\sqrt{\alpha} \tau) \operatorname{cn}(\sqrt{\alpha} \tau) \operatorname{dn}(\sqrt{\alpha} \tau)) \cos \left(\tau-\tau_{0}\right)\right. \\
\left.-\frac{\sqrt{\alpha} C_{1}}{T} \int_{0}^{T} \mathrm{~d} \tau \frac{2 \mathbf{K}\left(k_{1}\right)}{\sqrt{\alpha} \pi} \frac{\partial}{\partial \tau}\left(\operatorname{cn}^{2}(\sqrt{\alpha} \tau)\right)\right) \bar{\varphi}^{(n-1)} .
\end{gathered}
$$

This can be rewritten as

$$
\left\langle R^{(n)}\right\rangle=-\frac{16 k_{1}^{2} \mathbf{K}^{2}\left(k_{1}\right)}{\sqrt{\alpha} \pi^{2}} \cos \left(\tau_{0}\right) G_{1}(p, q) \bar{\varphi}^{(n-1)} .
$$

We refer the reader to Appendix Ef for more details on the evaluation of the integrals. The coefficient of $\bar{\varphi}^{(n-1)}$ is non-vanishing for $\tau_{0}$ chosen such that (2.21) is satisfied. Therefore it is possible to fix the initial conditions $\bar{\varphi}^{(n-1)}$ in such a way that one has $\left\langle F_{2}^{(n)}\right\rangle=0$ at all orders, thus completing the proof of the lemma.

Lemma 1 implies that the threshold values of the $p: q$ resonances are $\gamma(p / q)=C_{1}(p / q) \varepsilon$ for $p=1$ and $q$ even, with the constants $C_{1}(p / q)$ in Table 1 , while the threshold values of the other resonances are at least $O\left(\varepsilon^{2}\right)$.

\subsection{Rotations}

Similarly for the rotating scenario, again further details can be found in Appendix $\mathrm{D}$, the perturbed system can be written as

$$
\begin{aligned}
& \varphi^{\prime}=\frac{\pi \sqrt{\alpha}}{k_{2} \mathbf{K}\left(k_{2}\right)}+\frac{\varepsilon \pi k_{2}}{\sqrt{\alpha} \mathbf{K}\left(k_{2}\right)}\left[\frac{k_{2}^{2} \operatorname{sn}^{2}(\cdot) \operatorname{cn}^{2}(\cdot)}{1-k_{2}^{2}}-\frac{\mathbf{Z}(\cdot) \operatorname{sn}(\cdot) \operatorname{cn}(\cdot) \operatorname{dn}(\cdot)}{1-k_{2}^{2}}\right] \cos \left(\tau-\tau_{0}\right) \\
&-\frac{C_{1} \varepsilon \pi}{\mathbf{K}\left(k_{2}\right)}\left[\frac{k_{2}^{2} \operatorname{sn}(\cdot) \operatorname{cn}(\cdot) \operatorname{dn}(\cdot)}{1-k_{2}^{2}}-\frac{\mathbf{Z}(\cdot) \mathrm{dn}^{2}(\cdot)}{1-k_{2}^{2}}\right] \\
& I^{\prime}=\frac{4 \varepsilon \mathbf{K}\left(k_{2}\right)}{\pi} \cos \left(\tau-\tau_{0}\right) \operatorname{sn}(\cdot) \operatorname{cn}(\cdot) \operatorname{dn}(\cdot)-\frac{4 C_{1} \varepsilon \sqrt{\alpha} \mathbf{K}\left(k_{2}\right)}{\pi k_{2}} \operatorname{dn}^{2}(\cdot) .
\end{aligned}
$$

Here and throughout this subsection we set $(\cdot)=\left(\frac{\mathbf{K}\left(k_{2}\right)}{\pi} \varphi, k_{2}\right)$. In this scenario, the Wronskian matrix $W(\tau)$ can be written as in 2.14, with $\zeta^{(0)}$ given by, see Appendix B.

$$
\zeta(I)=-\frac{\pi^{2} \sqrt{\alpha}}{4 \mathbf{K}^{3}\left(k_{2}\right)}\left[\frac{\mathbf{E}\left(k_{2}\right)}{1-k_{2}^{2}}-\mathbf{K}\left(k_{2}\right)\right],
$$


for $k_{2}=k_{2}^{(0)}$. We again look for solutions $(\varphi(\tau), I(\tau))$ with period $T=2 \pi q=4 k_{2} \mathbf{K}\left(k_{1}\right) p / \sqrt{\alpha}$ corresponding to a resonance $p: q$, of the form $(2.7)$, the functions $\varphi^{(n)}(\tau)$ and $I^{(n)}(\tau)$ being defined as in (2.16), with $F_{1}^{(n)}(\tau)$ and $F_{2}^{(n)}(\tau)$ defined as

$$
\begin{gathered}
F_{1}^{(n)}(\tau)=\left[\frac{\pi \sqrt{\alpha}}{k_{2} \mathbf{K}\left(k_{2}\right)}-\zeta^{(0)} I\right]^{(n)}+\left[\frac { \pi k _ { 2 } } { \sqrt { \alpha } \mathbf { K } ( k _ { 2 } ) } \left[\frac{k_{2}^{2} \operatorname{sn}^{2}(\cdot) \mathrm{cn}^{2}(\cdot)}{1-k_{2}^{2}}\right.\right. \\
\left.-\frac{\mathbf{Z}(\cdot) \operatorname{sn}(\cdot) \operatorname{cn}(\cdot) \operatorname{dn}(\cdot)}{1-k_{2}^{2}}\right] \cos \left(\tau-\tau_{0}\right) \\
\left.-\frac{C_{1} \pi}{\mathbf{K}\left(k_{2}\right)}\left[\frac{k_{2}^{2} \operatorname{sn}(\cdot) \operatorname{cn}(\cdot)}{1-k_{2}^{2}}-\frac{\mathbf{Z}(\cdot) \operatorname{dn}(\cdot)}{1-k_{2}^{2}}\right]\right]^{(n-1)}, \\
F_{2}^{(n)}(\tau)=\left[\frac{4 \mathbf{K}\left(k_{2}\right)}{\pi} \cos \left(\tau-\tau_{0}\right) \operatorname{sn}(\cdot) \operatorname{cn}(\cdot) \operatorname{dn}(\cdot)-\frac{4 C_{1} \sqrt{\alpha} \mathbf{K}\left(k_{2}\right)}{\pi k_{2}} \operatorname{dn}^{2}(\cdot)\right]^{(n-1)} .
\end{gathered}
$$

The theory goes through exactly as previously shown for the case of libration and we must show that $\left\langle F_{2}^{(n)}\right\rangle=0$.

Lemma 2 Consider the series (2.7). If $p / q=1 / 2 m, m \in \mathbb{N}$, and $C_{1}$ is small enough, then it is possible to fix the initial conditions $\left(\bar{\varphi}^{(n)}, \bar{I}^{(n)}\right)$ in such a way that $\left\langle F_{2}^{(n)}\right\rangle=0$ for all $n \geq 1$. If $p / q \neq 1 / 2 m$ for all $m \in \mathbb{N}$ then $\left\langle F_{2}^{(n)}\right\rangle=0$ only when $C_{1}=0$.

Proof One has

$$
\begin{gathered}
F_{2}^{(1)}(\tau)=\frac{4 \mathbf{K}\left(k_{2}\right)}{\pi} \cos \left(\tau-\tau_{0}\right) \operatorname{sn}\left(\frac{\sqrt{\alpha}}{k_{2}} \tau, k_{2}\right) \operatorname{cn}\left(\frac{\sqrt{\alpha}}{k_{2}} \tau, k_{2}\right) \operatorname{dn}\left(\frac{\sqrt{\alpha}}{k_{2}} \tau, k_{2}\right) \\
-\frac{4 C_{1} \sqrt{\alpha} \mathbf{K}\left(k_{2}\right)}{\pi k_{2}} \operatorname{dn}^{2}\left(\frac{\sqrt{\alpha}}{k_{2}} \tau, k_{2}\right),
\end{gathered}
$$

with $k_{2}=k_{2}^{(0)}$ here and henceforth. Define, see Appendix E,

$$
\Delta:=\frac{\sqrt{\alpha}}{2 k_{2} \mathbf{K}\left(k_{2}\right)} \int_{0}^{2 k_{2} \mathbf{K}\left(k_{2}\right) / \sqrt{\alpha}} \mathrm{d} \tau \operatorname{dn}^{2}\left(\frac{\sqrt{\alpha}}{k_{2}} \tau, k_{2}\right)=\frac{1}{2 \mathbf{K}\left(k_{2}\right)} \mathbf{E}\left(2 \mathbf{K}\left(k_{2}\right), k_{2}\right) .
$$

and $\Gamma_{1}\left(\tau_{0} ; p, q\right):=\sin \left(\tau_{0}\right) G_{1}(p, q)$, where

$$
\begin{aligned}
G_{1}(p, q) & =\frac{1}{T} \int_{0}^{T} \mathrm{~d} \tau \operatorname{sn}\left(\frac{\sqrt{\alpha}}{k_{2}} \tau, k_{2}\right) \operatorname{cn}\left(\frac{\sqrt{\alpha}}{k_{2}} \tau, k_{2}\right) \operatorname{dn}\left(\frac{\sqrt{\alpha}}{k_{2}} \tau, k_{2}\right) \sin (\tau) \\
& =\frac{1}{4 \mathbf{K}\left(k_{2}\right) p} \int_{0}^{4 \mathbf{K}\left(k_{2}\right) p} \mathrm{~d} \tau \operatorname{sn}\left(\tau, k_{2}\right) \operatorname{cn}\left(\tau, k_{2}\right) \operatorname{dn}\left(\tau, k_{2}\right) \sin \left(k_{2} \tau / \sqrt{\alpha}\right),
\end{aligned}
$$

then use the resonance condition to set

$$
\sin \left(\frac{k_{2} \tau}{\sqrt{\alpha}}\right)=\sin \left(\frac{\pi \tau}{2 \mathbf{K}\left(k_{2}\right)} \frac{q}{p}\right) .
$$

On inspection of the above we see that $\Gamma_{1}\left(\tau_{0} ; p, q\right)$ can be calculated similarly to the case inside the separatrix. It follows that the same applies and $p / q=1 / 2 m$ for $m \in \mathbb{N}$. Then $\left\langle F_{2}^{(1)}\right\rangle=0$ if

$$
C_{1}=\frac{k_{2} \sin \left(\tau_{0}\right)}{\sqrt{\alpha} \Delta} G_{1}(p, q),
$$

which requires

$$
\left|C_{1}\right| \leq C_{1}(p / q):=\frac{k_{2}}{\sqrt{\alpha} \Delta} G_{1}(p, q) .
$$


Some values of the constants $C_{1}(p / q)$ for $\alpha=0.5$ are listed in Table 2

\begin{tabular}{|ccccc|}
\hline$q$ & $k_{2}$ & $G_{1}(1 / q)$ & $\Delta$ & $C_{1}(1 / q)$ \\
\hline 2 & 0.924397052341 & 0.156774 & 0.474414 & 0.432005 \\
4 & 0.998899257272 & 0.077612 & 0.225808 & 0.485542 \\
6 & 0.999986983601 & 0.051734 & 0.150063 & 0.487439 \\
8 & 0.999999846887 & 0.038800 & 0.112540 & 0.487577 \\
10 & 0.999999998199 & 0.031040 & 0.090032 & 0.487577 \\
12 & 0.999999999978 & 0.025867 & 0.075026 & 0.487577 \\
\hline
\end{tabular}

Table 2: Constants for the rotating attractors with $\alpha=0.5$.

For $n \geq 2$ one has

$$
F_{2}^{(n)}(\tau)=\left.\frac{4 \mathbf{K}\left(k_{2}\right)}{\pi} \frac{\partial}{\partial \varphi}\left(\cos \left(\tau-\tau_{0}\right) \operatorname{sn}(\cdot) \operatorname{cn}(\cdot) \operatorname{dn}(\cdot)-\sqrt{\alpha} C_{1} \operatorname{dn}^{2}(\cdot)\right)\right|_{\varphi=\varphi^{(0)}} \bar{\varphi}^{(n-1)}+R^{(n)}(\tau),
$$

where again $R^{(n)}(\tau)$ will be a suitable function which does not depend on $\bar{\varphi}^{(n-1)}$. Similarly to the case of libration, $\left\langle F_{2}^{(n)}\right\rangle=0$ if and only if

$$
\begin{aligned}
\left\langle R^{(n)}\right\rangle & =-\frac{4 \mathbf{K}\left(k_{2}\right)}{\pi}\left(\frac{1}{T} \int_{0}^{T} \frac{k_{2} \mathbf{K}\left(k_{1}\right)}{\sqrt{\alpha} \pi} \frac{\partial}{\partial \tau}(\operatorname{sn}(\sqrt{\alpha} \tau) \operatorname{cn}(\sqrt{\alpha} \tau) \operatorname{dn}(\sqrt{\alpha} \tau)) \cos \left(\tau-\tau_{0}\right) \mathrm{d} \tau\right. \\
& \left.-\frac{\sqrt{\alpha} C_{1}}{T} \int_{0}^{T} \frac{k_{2} \mathbf{K}\left(k_{1}\right)}{\sqrt{\alpha} \pi} \frac{\partial}{\partial \tau}\left(\operatorname{dn}^{2}(\sqrt{\alpha} \tau)\right) \mathrm{d} \tau\right) \bar{\varphi}^{(n-1)}=-\frac{4 k_{2} \mathbf{K}^{2}\left(k_{2}\right)}{\sqrt{\alpha} \pi^{2}} \cos \left(\tau_{0}\right) G_{1}(p, q) \bar{\varphi}^{(n-1)}
\end{aligned}
$$

so that the coefficient of $\bar{\varphi}^{(n-1)}$ turns out to be non-vanishing for $\tau_{0}$ chosen such that equation (2.24) is satisfied. Therefore it is possible to fix the initial conditions $\bar{\varphi}^{(n-1)}$ in such a way that one has $\left\langle F_{2}^{(n)}(\tau)\right\rangle=0$ at all orders, thus completing the proof.

Lemma 2 implies that the threshold values of the $p: q$ resonances are $\gamma(p / q)=C_{1}(p / q) \varepsilon$ for $p=1$ and $q$ even, with the constants $C_{1}(p / q)$ in Table2, while the threshold values of the other resonances are at least $O\left(\varepsilon^{2}\right)$.

Note, in Tables 1 and 2 it is apparent that, for $\alpha=0.5$, increasing $q$ causes the value of $C_{1}(1 / q)$ to converge to 0.487577 in both cases. However this does not mean that for $\gamma<0.487577 \varepsilon$ there are infinitely many attracting solutions with increasing period: this would be a counter-example to Palis' conjecture! The explanation for this seeming paradox is as follows: As $q$ increases the solutions move closer and closer to the separatrix (this can be seen by the corresponding values of $k_{1}$ and $k_{2}$ ), where the power series expansions 2.7 for the solutions $I(\tau)$ and $\varphi(\tau)$ which were constructed with perturbation theory converge only for very small values of $\varepsilon$ : the larger $q$, the smaller must be $\varepsilon$. In particular, for any fixed $\varepsilon$ there is only a finite number of periodic solutions which can be studied by perturbation theory. In particular, for the chosen parameters the only periodic solution corresponds to the resonance 1:2 inside the separatrix. We also note that the above analysis applies only to periodic attractors with $p=1$ and $q$ even. However we shall see that the numerical simulations provide also rotating attractors with period $2 \pi$, that is the same period as the forcing: we expect that continuing the analysis to second order and writing $\gamma=C_{2} \varepsilon^{2}$, see [1, would give the threshold values for these periodic attractors.

\section{Numerics for the downwards pendulum}

We shall investigate the system (1.3) in the same region of phase space used in [5], namely $\theta \in[-\pi, \pi]$, $\theta^{\prime} \in[-4,4]$ and calculate the relative areas of the basins of attraction, that is the percentage of phase space they cover relative to this region. 
Throughout this Section we fix the parameters $\alpha=0.5$ and $\beta=0.1$. These parameter values, also investigated in [5], correspond to a stable region of the stability tongues for the system linearised around $\theta=0$, see $[22$, so that the downwards configuration is stable. The chosen values for the damping coefficient span values between $\gamma=0.002$ and 0.06 , of which only $\gamma=0.03$ was previously investigated in [5]. For some values of $\gamma$, the system exhibits three non-fixed-point attractors, examples of which are shown in Figure 1, as well as the downwards fixed point attractor. Here and henceforth, for brevity, we shall say that a solution has period $n$ if it comes back to its initial value after $n$ periods of the forcing. Of course the upwards fixed point also exists as a solution to the system, however it is unstable and thus does not attract any non-zero measure subset of phase space. It can also be seen from Figure 1 that the attractive solutions are near the separatrix of the unperturbed system: this is evident as the curves described by the two rotating attractors are close to that of the oscillating attractor and the separatrix lies between them. This observation confirms the reasoning as to why the computation of the threshold values can only produce valid results for the period 2 oscillating attractor (see the conclusive remarks in Section 2).

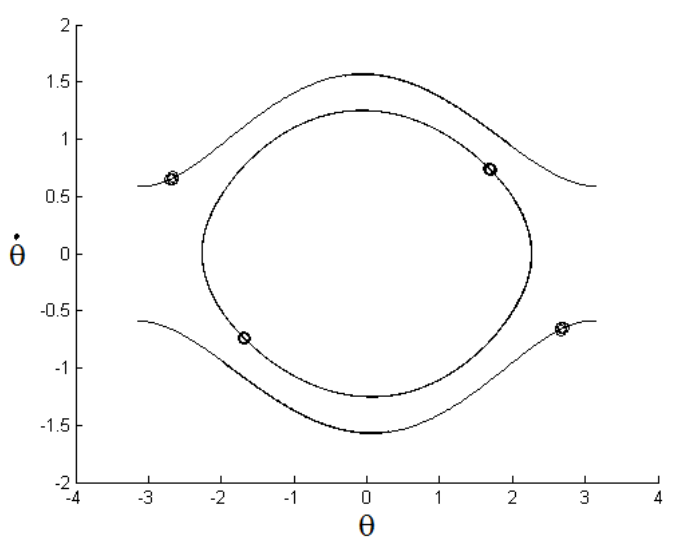

Figure 1: Attracting solutions for the system 1.3 with $\alpha=0.5, \beta=0.1$ and $\gamma=0.02$, namely two period 1 rotations and one period 2 solution which oscillates about the downwards fixed point. Periods can be deduced by circles corresponding to the Poincaré map.

For $\gamma<\bar{\gamma}_{0}$, for a suitable $\bar{\gamma}_{0} \in(0.4,0.5)$, the system has three periodic attractors, in addition to the downwards fixed point: one oscillating and two rotating attractors. For $\gamma \geq \bar{\gamma}_{0}$ the two rotating attractors no longer exist, leaving just the oscillatory attractor and the fixed point. The basins of attraction for $\gamma=0.02,0.03,0.04$ and 0.05 are shown in Figure 2, from which we can see that the entire phase space is covered: this suggests that no other attractors exist, at least for the values of the parameters considered. The corresponding relative areas, as estimated by the numerical simulations, are given in Table 3 and plotted in Figure 3 . The relative areas of the basins of attraction for positive and negative rotations have been listed in the same column: numerical simulations found a difference in size no greater than $10^{-2} \%$ and, due to the symmetries of the system, it is expected that this difference is numerically induced by the selection of initial conditions The basins of attraction were estimated using numerical simulations with 600000 random initial conditions in phase space. More notes on the numerics used can be found in Section 5.

It can be seen that the results in Table 3 are in agreement with the calculations for the threshold value for the period 2 oscillatory attractor. The calculations in Appendix A predict that, for the chosen values $\alpha=0.5$ and $\beta=0.1$, taking $\gamma>\bar{\gamma}_{1} \approx 0.1021$ ensures for the origin to capture a full measure set of initial conditions. From Table 3 a stronger result emerges numerically: the fixed point attracts the full phase space, up to a zero-measure set, for $\gamma \geq \bar{\gamma}_{2} \approx 0.06$. Upon comparing results in Table 3, we see that, essentially, the basin of attraction for the fixed point becomes smaller with an increase in $\gamma$, up to approximately 0.035, after which it grows again. Similarly, by increasing the value of $\gamma$, the basins of attraction of the oscillating and rotating solutions attractors increase initially, up to some value (about 0.025 and 0.035 , respectively), after which they become smaller. 
Furthermore, the variations of the relative areas of the basins of attraction are never monotonic, as one observes slight oscillations for small variations of $\gamma$. These features seem contrary to systems such as the cubic oscillator, where decreasing dissipation seems to cause the relative area of the basin of attraction of the fixed point to decrease monotonically, while the basins of attraction of the periodic attractors reach a maximum value, after which their relative areas slightly decrease, see for instance Table III in [3]. We note, however, that a more detailed investigation shows that oscillations occur also in the case of the cubic oscillator. This was already observed for some values of the parameters (see Table IX in [3]), but the phenomenon can also be observed for the parameter values of Table III, simply by considering smaller changes of the value of $\gamma$ with respect to the values in [3]. For instance, by varying slightly $\gamma$ around 0.0005 (see Table III in [3] for notations), one finds for the main attractors the relative areas in Table 4

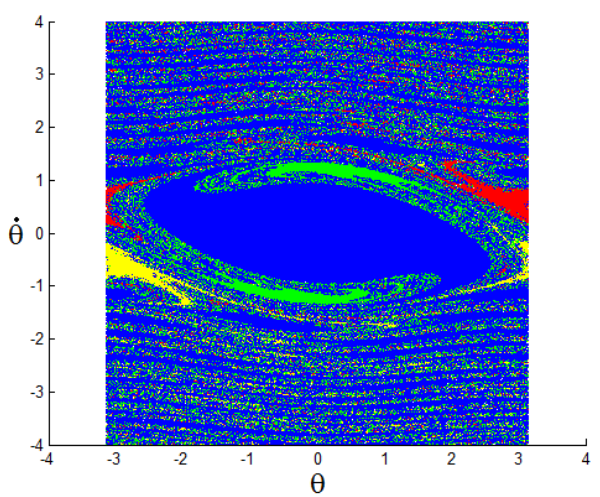

(a)

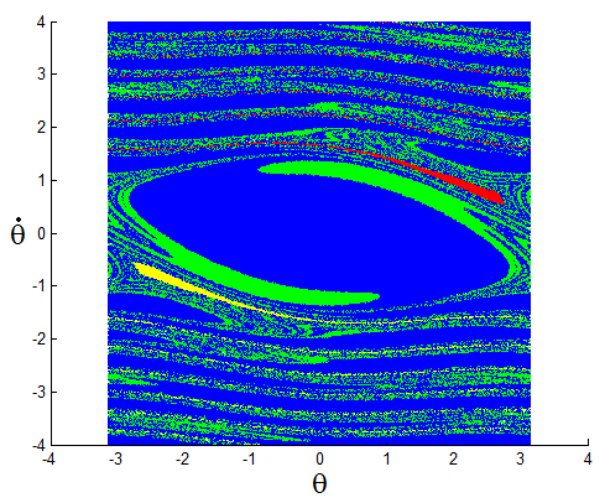

(c)

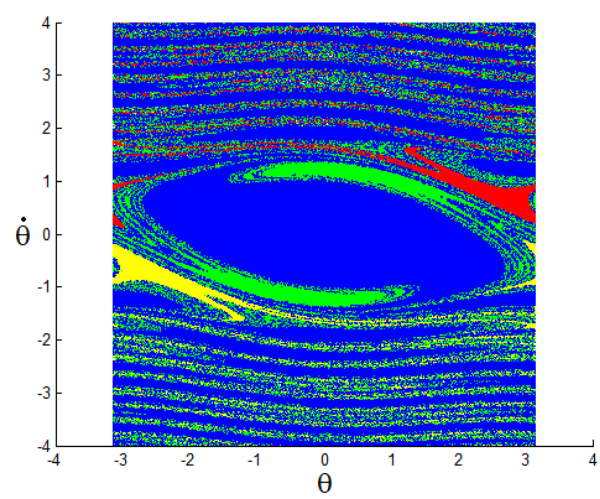

(b)

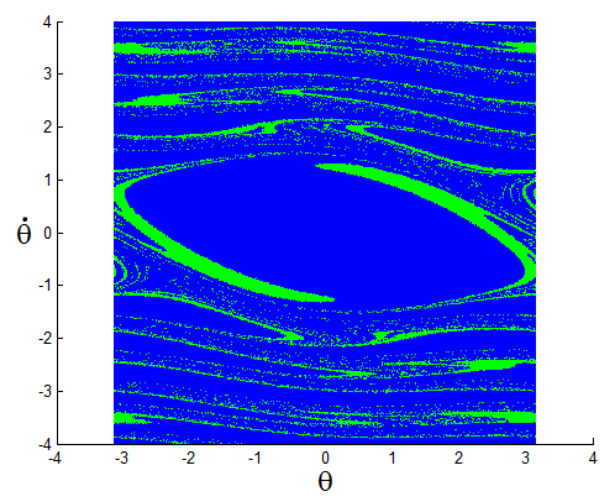

(d)

Figure 2: Basins of attraction for the system (1.3) with $\alpha=0.5, \beta=0.1$ and (a) $\gamma=0.02$, (b) $\gamma=0.03$, (c) $\gamma=0.04$ and (d) $\gamma=0.05$. The fixed point (FP) is shown in blue, the positive and negative rotating solutions (PR and NR) are shown in red and yellow, respectively. and the oscillating solution (OSC) in green.

In conclusion, for the pendulum, apart from small oscillations, by decreasing the value of $\gamma$ from 0.06 to 0.002 , the basins of attraction of the periodic attractors, after reaching a maximum value, becomes smaller. A similar phenomenon occurs also in the cubic oscillator (albeit less pronounced). However, a new feature of the pendulum, with respect to the cubic oscillator, is that the basin of attraction of the origin after reaching a minimum value increases again: the increase seems to be too large to be ascribed simply to an oscillation, even though this cannot be excluded. In the case of the cubic oscillator the slight decrease of the sizes of the basins of attraction of the periodic attractors was due essentially to the appearance of new attractors and their corresponding basins 
of attraction. It would be interesting to investigate further, in the case of the pendulum, how the basins of attractions, in particular that of the fixed point, change by taking smaller and smaller values of $\gamma$. We intend to come back to this in the future [36].

\begin{tabular}{|c|c|c|c|c|}
\hline & \multicolumn{3}{|c|}{ Basin of attraction \% } \\
\hline & & FP & $\mathrm{PR} / \mathrm{NR}$ & OSC \\
\hline \multirow{14}{*}{$\gamma$} & 0.0020 & 84.57 & 3.35 & 8.73 \\
\hline & 0.0050 & 79.91 & 3.88 & 12.32 \\
\hline & 0.0100 & 72.24 & 4.60 & 18.57 \\
\hline & 0.0200 & 71.95 & 4.57 & 18.90 \\
\hline & 0.0230 & 70.73 & 5.18 & 18.90 \\
\hline & 0.0250 & 69.28 & 5.19 & 20.35 \\
\hline & 0.0300 & 69.94 & 4.42 & 21.23 \\
\hline & 0.0330 & 68.92 & 3.75 & 23.59 \\
\hline & 0.0350 & 68.77 & 3.16 & 24.90 \\
\hline & 0.0400 & 73.84 & 1.42 & 23.32 \\
\hline & 0.0500 & 85.61 & 0.00 & 14.39 \\
\hline & 0.0590 & 96.96 & 0.00 & 3.04 \\
\hline & 0.0597 & 98.59 & 0.00 & 1.41 \\
\hline & 0.0600 & 100.00 & 0.00 & 0.00 \\
\hline
\end{tabular}

Table 3: Relative areas of the basins of attraction with $\alpha=0.5, \beta=0.1$ and constant $\gamma$.
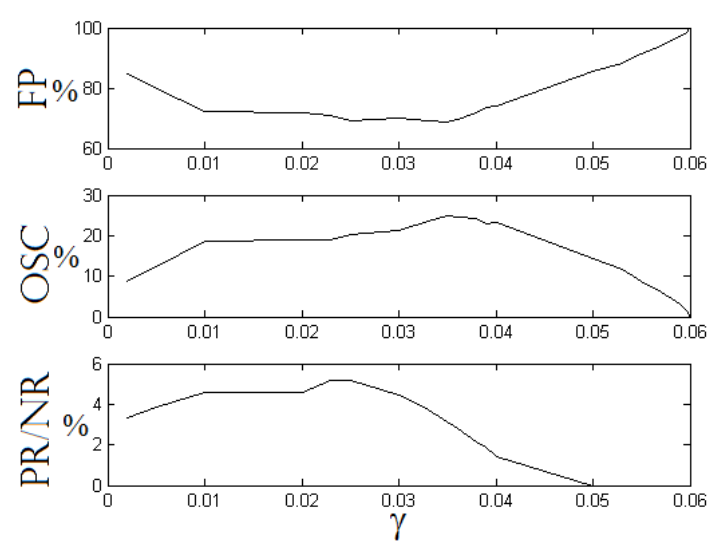

Figure 3: Plot of the relative areas of the basins of attraction with constant $\gamma$ as per Table 3

\begin{tabular}{|c|c|c|c|c|c|c|c|c|c|}
\cline { 3 - 10 } \multicolumn{2}{c|}{} & \multicolumn{8}{c|}{ Basin of attraction \% } \\
\cline { 3 - 10 } \multicolumn{1}{c|}{} & 0 & $1 / 2$ & $1 / 4$ & $1 \mathrm{a}$ & $1 \mathrm{~b}$ & $1 / 6$ & $3 \mathrm{a}$ & $3 \mathrm{~b}$ \\
\hline \multirow{4}{*}{$\gamma$} & 0.00052 & 39.03 & 41.73 & 14.72 & 1.22 & 1.22 & 1.59 & 0.25 & 0.25 \\
\cline { 2 - 10 } & 0.00051 & 39.73 & 41.70 & 13.88 & 1.24 & 1.24 & 1.66 & 0.27 & 0.27 \\
\cline { 2 - 10 } & 0.00050 & 38.72 & 41.85 & 14.65 & 1.28 & 1.28 & 1.65 & 0.29 & 0.29 \\
\cline { 2 - 10 } & 0.00049 & 39.26 & 41.96 & 13.81 & 1.29 & 1.29 & 1.77 & 0.27 & 0.32 \\
\cline { 2 - 10 } & 0.00048 & 38.48 & 41.87 & 14.60 & 1.30 & 1.30 & 1.75 & 0.34 & 0.34 \\
\hline
\end{tabular}

Table 4: Relative areas of the basins of attraction of the main attractors for the system $\ddot{x}+(1+\varepsilon \cos t) x^{3}+\gamma \dot{x}=0$, with $\varepsilon=0.1$ and $\gamma$ around 0.0005 . The basins of attraction were estimated using numerical simulations with 1000000 random initial conditions in the square $[-1,1] \times[-1,1]$ in phase space.

\subsection{Increasing dissipation}

In this section we shall investigate the case where dissipation increases with time, up to a time $T_{0}$, after which it remains constant. We will consider a linear increase in dissipation from a value $\gamma_{0}$ at time $t=0$ up to $\gamma_{1}$ at time $T_{0}$, that is (see Figure 4)

$$
\gamma=\gamma(t)= \begin{cases}\gamma_{0}+\left(\gamma_{1}-\gamma_{0}\right) \frac{\tau}{T_{0}}, & 0 \leq \tau<T_{0} \\ \gamma_{1}, & T_{0} \leq \tau\end{cases}
$$

Although this is a greatly simplified model of what might take place in reality, it serves the purpose of demonstrating the significant effects of initially non-constant dissipation on the final basins of attraction. Below we will consider explicitly the cases $\gamma_{0}=0.2$ and $\gamma_{1}=0.3,0.4$ and 0.5.

As previously mentioned, we expect that increasing the value of $T_{0}$ results in the relative areas of the basins of attraction moving along the curves plotted for constant $\gamma$. The movement along these curves starts at $\gamma_{1}$ and goes towards $\gamma_{0}$. In particular, any values of the relative area of a basin of attraction for constant values of the damping coefficient between $\gamma_{1}$ and $\gamma_{0}$ are traced as the value of $T_{0}$ varies for time-dependent dissipation. This movement along the curve is not linear with 
the value of $T_{0}$ but asymptotic, with the relative area of the basin of attraction tending towards the value at $\gamma=\gamma_{0}$ as $T_{0} \rightarrow \infty$, providing the attractors existing at $\gamma=\gamma_{0}$ also persist at $\gamma_{1}$. When the attractors which persist at $\gamma=\gamma_{1}$ are a proper subset of those which exist at $\gamma_{0}$, we expect the persisting attractors to absorb the remaining phase space left by the attractors which have disappeared: thus their basins of attraction should be greater than or equal to those at $\gamma=\gamma_{0}$. For the values of the parameters in the chosen range, only these two cases may occur as the solutions which exist for $\gamma=\gamma_{1}$ also exist at $\gamma=\gamma_{0}$, see Table 3

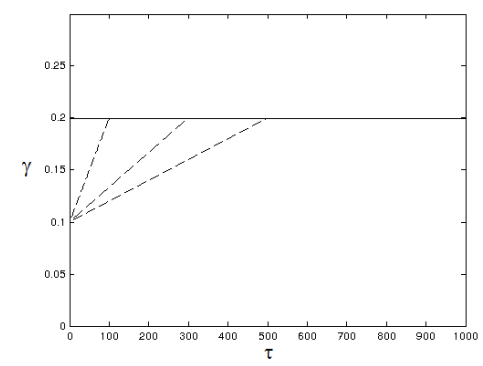

Figure 4: Plot of equation 3.1 with $\gamma_{0}=0.1, \gamma_{1}=0.2$ and varying $T_{0}$.

The results in Tables 5,6 and 7 are in agreement with the expectations above. It can be seen from Tables 5 and 6 that the relative areas of the basins of attraction trace those of constant $\gamma$. In particular, the relative area of the basins of attraction of the rotating attractors tends towards that at $\gamma=\gamma_{0}$ from above, despite having a smaller basin of attraction for the chosen values of $\gamma_{1}$. More precisely, the longer $T_{0}$, the closer is the relative area of the basin of attraction to the value it has at $\gamma=\gamma_{0}$. However, the convergence to the asymptotic value is rather slow: for instance in Table 5 . even $T_{0}=2000$ is not enough to reach the values corresponding to $\gamma=0.02$. The simulations for time varying dissipation have in general taken 300000 or 400000 initial conditions in phase space. In some cases more points were used for additional accuracy.

\begin{tabular}{|c|c|c|c|c|}
\hline & \multicolumn{3}{|c|}{ Basin of Attraction \% } \\
\hline & & $\mathrm{FP}$ & $\mathrm{PR} / \mathrm{NR}$ & $\mathrm{OSC}$ \\
\hline \multirow{9}{*}{$T_{0}$} & 0 & 69.94 & 4.42 & 21.23 \\
\hline & 25 & 69.80 & 4.42 & 21.36 \\
\hline & 50 & 69.57 & 4.45 & 21.52 \\
\hline & 75 & 69.40 & 4.64 & 21.33 \\
\hline & 100 & 68.84 & 4.85 & 21.47 \\
\hline & 200 & 68.82 & 5.10 & 20.99 \\
\hline & 500 & 69.86 & 5.17 & 19.80 \\
\hline & 1000 & 70.65 & 5.18 & 18.99 \\
\hline & 2000 & 71.17 & 5.11 & 18.61 \\
\hline
\end{tabular}

Table 5: Relative areas of the basins of attraction with $\gamma_{0}=0.02, \gamma_{1}=0.03$ and $T_{0}$ varying.
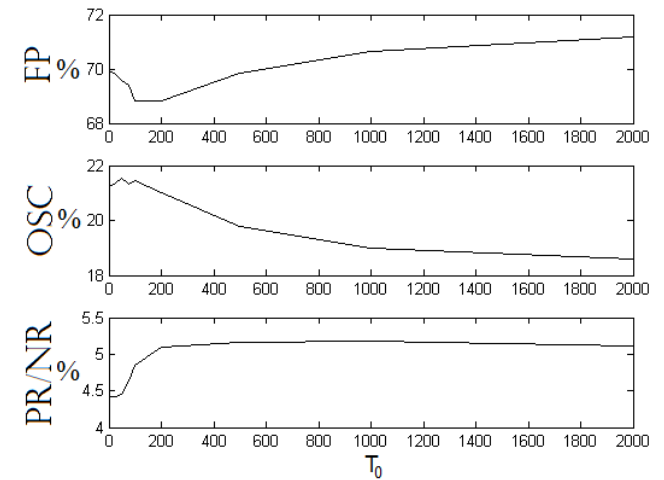

Figure 5: Plot of the relative areas of the basins of attraction as per Table 5

In Table 7, we see that for $\gamma_{0}=0.02$ and $\gamma_{1}=0.05$ only two attractors are present: indeed the oscillating attractors no longer exist for $\gamma=0.05$. Hence, when $\gamma(t)$ increases and crosses the value at which those attractors disappear, all the trajectories that up to this time were converging to them, will fall into the basins of attraction of the persisting attractors, that is the fixed point and the oscillating solution. In particular the corresponding basins of attraction will acquire relative areas larger than those they have at constant $\gamma=\gamma_{0}$, because of the absorption of all these trajectories. It is difficult to predict how such trajectories are distributed among the persisting attractors. In 
the case of Table 7 they seem to be attracted slightly more by the fixed point, even though the percentage increase is larger for the oscillating solution.

\begin{tabular}{|c|c|c|c|c|}
\cline { 3 - 5 } \multicolumn{2}{c|}{} & \multicolumn{3}{c|}{ Basin of Attraction \% } \\
\cline { 3 - 5 } \multicolumn{1}{c|}{} & FP & PR/NR & OSC \\
\hline \multirow{5}{*}{$T_{0}$} & 0 & 73.84 & 1.42 & 23.32 \\
\cline { 2 - 5 } & 25 & 73.66 & 1.44 & 23.45 \\
\cline { 2 - 5 } & 50 & 73.37 & 1.50 & 23.63 \\
\cline { 2 - 5 } & 75 & 72.15 & 2.22 & 23.41 \\
\cline { 2 - 5 } & 100 & 68.69 & 3.50 & 24.31 \\
\cline { 2 - 5 } & 200 & 67.46 & 4.76 & 23.03 \\
\cline { 2 - 5 } & 500 & 69.05 & 5.02 & 20.92 \\
\cline { 2 - 5 } & 1000 & 69.85 & 5.17 & 19.81 \\
\cline { 2 - 5 } & 2000 & 70.63 & 5.18 & 19.02 \\
\hline
\end{tabular}

Table 6: Relative areas of the basins of attraction with $\gamma_{0}=0.02, \gamma_{1}=0.04$ and $T_{0}$ varying.

\begin{tabular}{|c|c|c|c|}
\cline { 3 - 4 } \multicolumn{2}{c|}{} & \multicolumn{2}{c|}{ Basin of Attraction \% } \\
\cline { 3 - 4 } \multicolumn{1}{c|}{} & FP & OSC \\
\hline \multirow{5}{*}{$T_{0}$} & 0 & 85.61 & 14.39 \\
\hline & 25 & 86.01 & 13.99 \\
\cline { 2 - 4 } & 50 & 86.18 & 13.82 \\
\cline { 2 - 4 } & 75 & 84.19 & 15.87 \\
\cline { 2 - 4 } & 100 & 80.42 & 19.58 \\
\cline { 2 - 4 } & 200 & 75.47 & 24.53 \\
\cline { 2 - 4 } & 500 & 77.95 & 22.06 \\
\cline { 2 - 4 } & 1000 & 77.55 & 22.45 \\
\cline { 2 - 4 } & 1500 & 78.03 & 21.97 \\
\hline
\end{tabular}

Table 7: Relative areas of the basins of attraction with $\gamma_{0}=0.02, \gamma_{1}=0.05$ and $T_{0}$ varying.

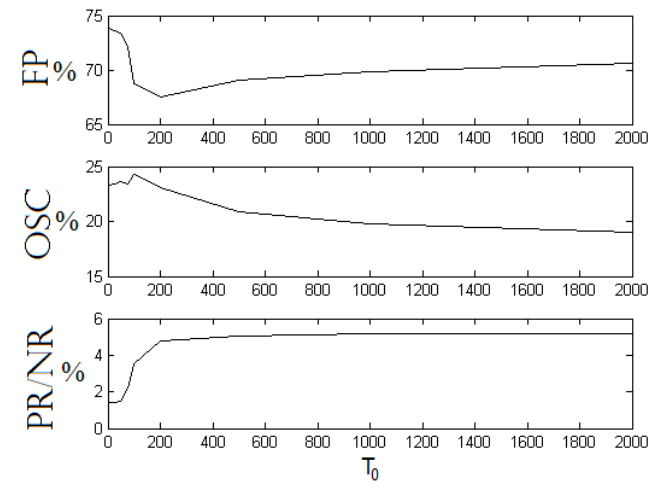

Figure 6: Plot of the relative areas of the basins of attraction as per Table 6
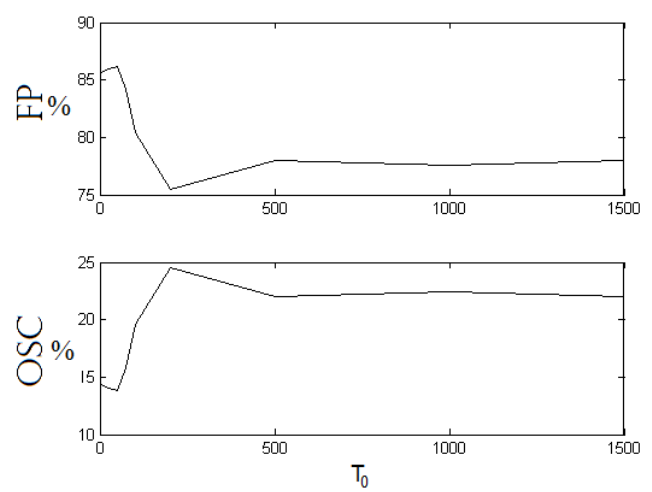

Figure 7: Plot of the relative areas of the basins of attraction as per Table 7

\subsection{Decreasing dissipation}

In this section we conversely look at the damping coefficient decreasing from some value $\gamma_{0}>\gamma_{1}$, with different rates of decrease, see Figure 8. We will consider the cases $\gamma_{0}=0.04$ and $\gamma_{1}=0.02$, $\gamma_{0}=0.04$ and $\gamma_{1}=0.03, \gamma_{0}=0.05$ and $\gamma_{1}=0.02$.

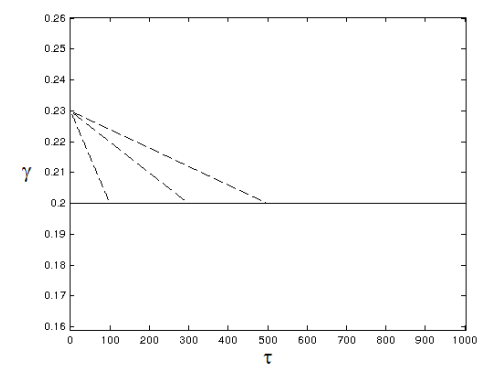

Figure 8: Plot of equation 3.1 with $\gamma_{0}=0.23, \gamma_{1}=0.2$ and varying $T_{0}$. 
In this situation it is possible that more attractors exist at $\gamma_{1}$ than at $\gamma_{0}$, see Table 3 . We again expect that increasing $T_{0}$ causes the relative areas of the basins of attraction to tend towards those at $\gamma_{0}$. The result of this is that solutions which do not exist at $\gamma_{0}$ will attract less and less of the phase space as $T_{0}$ increases, and for $T_{0}$ large enough their basins of attraction will tend to zero.

\begin{tabular}{|c|c|c|c|c|}
\hline & \multicolumn{3}{|c|}{ Basin of Attraction \% } \\
\hline & & $\mathrm{FP}$ & $\mathrm{PR} / \mathrm{NR}$ & $\mathrm{OSC}$ \\
\hline \multirow{9}{*}{$T_{0}$} & 0 & 71.95 & 4.57 & 18.90 \\
\hline & 25 & 71.85 & 4.60 & 18.94 \\
\hline & 50 & 72.36 & 4.48 & 18.69 \\
\hline & 75 & 73.64 & 4.43 & 17.51 \\
\hline & 100 & 74.10 & 4.32 & 17.27 \\
\hline & 200 & 72.31 & 2.99 & 21.71 \\
\hline & 500 & 71.51 & 2.09 & 24.31 \\
\hline & 1000 & 72.61 & 1.79 & 23.81 \\
\hline & 2000 & 73.11 & 1.63 & 23.64 \\
\hline
\end{tabular}

Table 8: Relative areas of the basins of attraction with $\gamma_{0}=0.04, \gamma_{1}=0.02$ and $T_{0}$ varying.

\begin{tabular}{|c|c|c|c|c|}
\cline { 3 - 5 } \multicolumn{2}{c|}{} & \multicolumn{3}{c|}{ Basin of Attraction \% } \\
\cline { 3 - 5 } \multicolumn{1}{c|}{} & FP & PR/NR & OSC \\
\hline \multirow{5}{*}{$T_{0}$} & 0 & 69.94 & 4.42 & 21.23 \\
\cline { 2 - 5 } & 25 & 69.73 & 4.50 & 21.28 \\
\cline { 2 - 5 } & 50 & 70.78 & 4.23 & 20.77 \\
\cline { 2 - 5 } & 75 & 72.03 & 3.45 & 21.07 \\
\cline { 2 - 5 } & 100 & 71.77 & 2.95 & 22.33 \\
\cline { 2 - 5 } & 500 & 72.61 & 1.79 & 23.81 \\
\cline { 2 - 5 } & 1000 & 73.11 & 1.63 & 23.64 \\
\hline
\end{tabular}

Table 9: Relative areas of the basins of attraction with $\gamma_{0}=0.04, \gamma_{1}=0.03$ and $T_{0}$ varying.

\begin{tabular}{|c|c|c|c|c|}
\cline { 3 - 5 } \multicolumn{2}{c|}{} & \multicolumn{3}{|c|}{ Basin of Attraction \% } \\
\cline { 3 - 5 } \multicolumn{1}{c|}{} & FP & PR/NR & OSC \\
\hline \multirow{4}{*}{$T_{0}$} & 0 & 71.95 & 4.57 & 18.90 \\
\cline { 2 - 5 } & 25 & 72.13 & 4.58 & 18.72 \\
\cline { 2 - 5 } & 50 & 72.97 & 4.24 & 18.55 \\
\cline { 2 - 5 } & 75 & 76.14 & 3.15 & 17.56 \\
\cline { 2 - 5 } & 100 & 77.02 & 2.18 & 18.62 \\
\cline { 2 - 5 } & 200 & 77.71 & 0.31 & 21.67 \\
\cline { 2 - 5 } & 500 & 81.94 & 0.00 & 18.06 \\
\hline
\end{tabular}

Table 10: Relative areas of the basins of attraction with $\gamma_{0}=0.05, \gamma_{1}=0.02$ and $T_{0}$ varying.
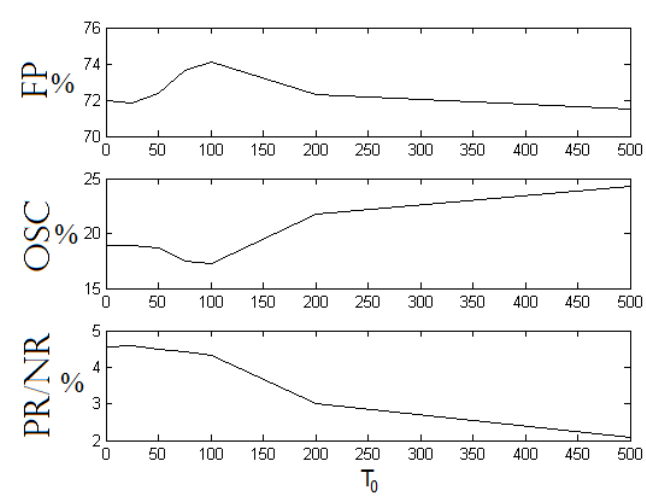

Figure 9: Plot of the relative areas of the basins of attraction as per Table 8
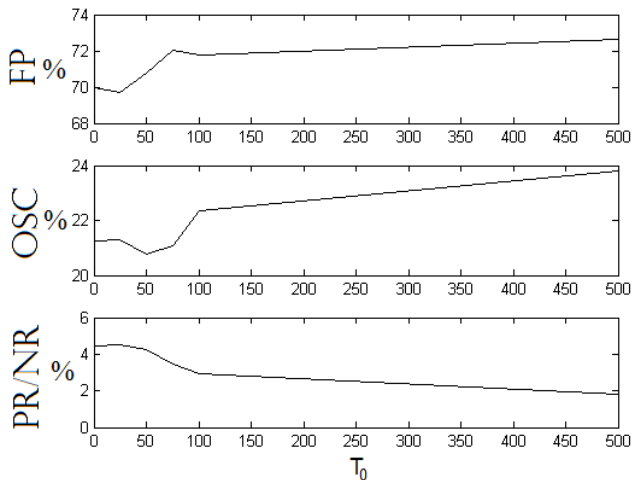

Figure 10: Plot of the relative areas of the basins of attraction as per Table 9
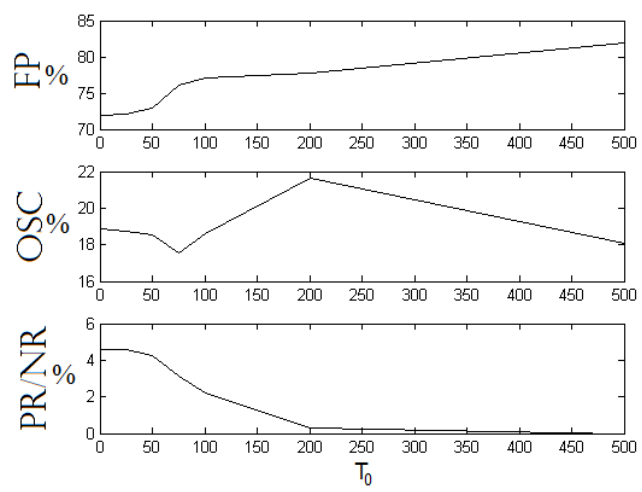

Figure 11: Plot of the relative areas of the basins of attraction as per Table 10 
Tables 8 and 9 illustrate cases in which the system admits the same set of attractors for both values $\gamma_{0}$ and $\gamma_{1}$ of the damping coefficient. An example of what happens when an attractor exists at $\gamma_{1}$ but not at $\gamma_{0}$ can be seen in the results of Table 10 , where $\gamma(t)$ varies from 0.05 to 0.02 . As the damping coefficient starts off at a larger value, then decreases to some smaller value, we also expect the change in the basins of attraction to happen over shorter values of $T_{0}$. The reasoning for this is simply that larger values of dissipation cause trajectories to move onto attractors in less time. Increasing $T_{0}$ results in the system remaining at higher values of dissipation for more time and thus trajectories land on the attractors in less time.

\section{Numerics for the inverted pendulum}

The upwards fixed point of the inverted pendulum can be made stable for large values of $\beta$, i.e when the amplitude of the oscillations is large relative to the length of the pendulum. In this section we numerically investigate the system 1.3 for parameter values for which this happens. For simplicity, as mentioned in the introduction, we refer to this case as the inverted pendulum. It can be more convenient to set $x=\pi+\xi$, so as to centre the origin at the upwards position of the pendulum. Then the equations of motion become

$$
\begin{gathered}
\xi^{\prime \prime}+f(\tau) \sin \xi+\gamma \xi^{\prime}=0, \quad f(\tau)=-(\alpha+\beta \cos \tau), \\
\alpha=\frac{g}{\ell \omega^{2}}, \quad \beta=\frac{b}{\ell}, \quad \tau=\omega t .
\end{gathered}
$$

The difference between equations $(1.3)$ and $(4.1)$ is that here the parameter $\alpha$ has changed sign.

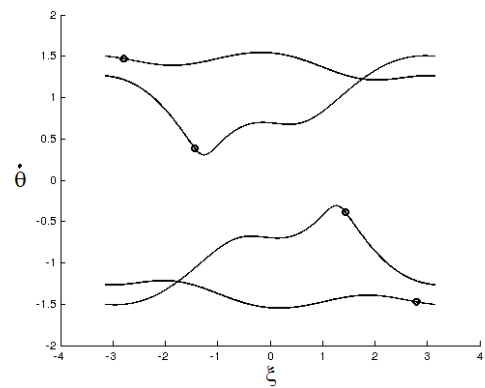

(a)

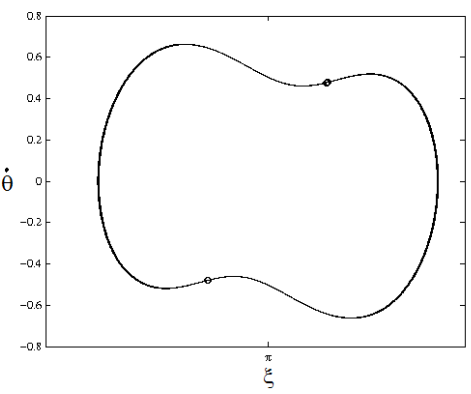

(c)

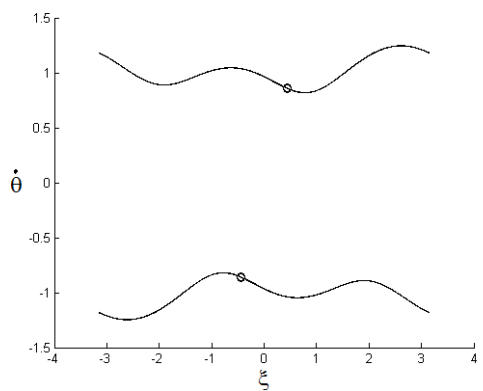

(b)

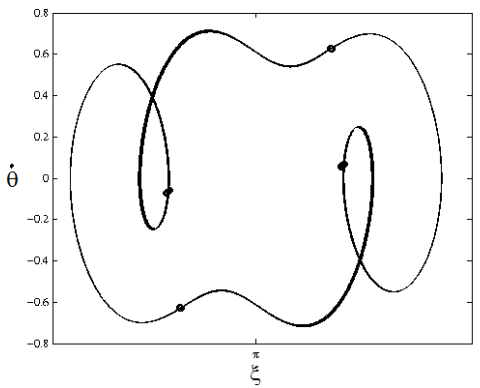

(d)

Figure 12: Attracting solutions for the system 4.1 with $\alpha=0.1$ and $\beta=0.545$. Figure (a) shows an example of the positive and negative rotating attractors with period 2, taken for $\gamma=0.05$. Figure (b) shows the positive and negative attractors with period 1 when $\gamma=0.23$. Figures (c) and (d) show the oscillatory attractors with periods 2 and 4 respectively when $\gamma$ is taken equal to 0.2725 . These solutions oscillate about the downwards fixed point $\xi=\pi$ and the axis has been shifted to show a connected curve in phase space. The period of each solution can be deduced from the circles corresponding to the Poincaré map. 
The stability of the upwards fixed point creates interesting dynamics to study numerically, however it means that the system is no longer a perturbation of the simple pendulum system. This in turn has the result that the analysis in Section 2 to compute the thresholds of friction cannot be applied. However, we shall see that the very idea that attractors have a threshold value below which they always exist does not apply to the inverted pendulum: both increasing and decreasing the damping coefficient can create and destroy solutions.

For numerical simulations of the inverted pendulum throughout we shall take parameters $\alpha=0.1$ and $\beta=0.545$, which are within the stable regime for the upwards position. For these parameter values the function $f(\tau)$ changes sign. As such, the analysis in Appendix A cannot be applied. Again these particular parameter values were also investigated in [5], but with a small value for the damping coefficient, that is $\gamma=0.08$, where only three attractors appeared in the system: the upwards fixed point and the left and right rotating solutions. We have opted to focus on larger dissipation because the range of values considered for $\gamma$ allows us to incorporate already a a wide variety of dynamics, in which remarkable phenomena occur, and, at the same time, larger values of $\gamma$ are better suited to numerical simulation because of the shorter integration times. We note that, for the values of the parameters chosen, no strange attractors arise: numerically, besides the fixed points, only periodic attractors are found.

For constant dissipation we provide results for $\gamma \in[0.05,0.6]$. These values of $\gamma$ are considered to correspond to large dissipation, however non-fixed-point solutions still persist due to the large coefficient $\beta$ of the forcing term. Some examples of the persisting non-fixed-point solutions can be seen in Figure 12 of course, the exact form of the curves depends on the particular choices of $\gamma$. For $\gamma$ varying in the range considered the following attractors arise (we follow the same convention as in Section 3 when saying that a solution has period $n$ ): the upwards fixed point (FP), the downwards fixed point (DFP), a positively rotating period 1 solution (PR), a negatively rotating period 1 solution (NR), a positively rotating period 2 solution (PR2), a negatively rotating period 2 solution (NR2), an oscillating period 2 solution (DO2) and an oscillating period 4 solution (DO4). However, as we will see, the solution DO2 deserves a separate, more detailed discussion.

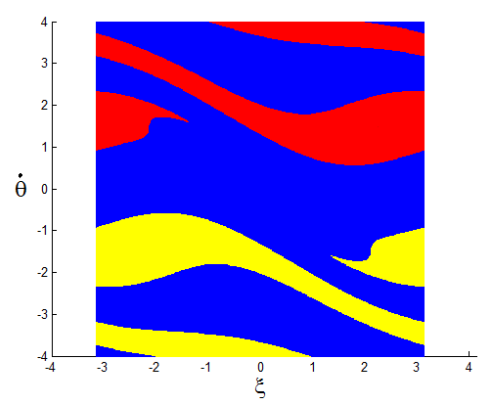

(a)

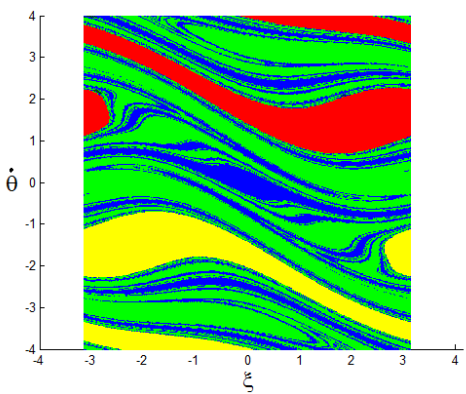

(b)

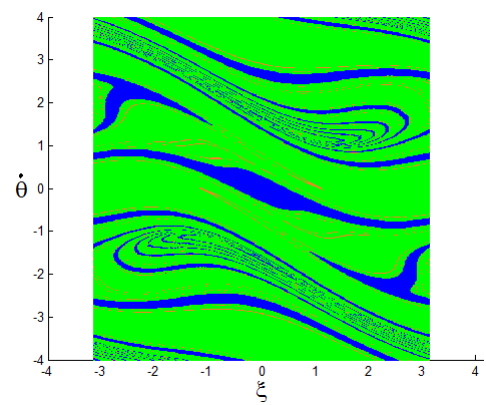

(c)

Figure 13: Basins of attraction for constant dissipation with $\gamma=0.2,0.23$ and 0.2725 from left to right respectively. The fixed point (FP) is shown in blue, the positively rotating solution (PR) in red, the negatively rotating solution (NR) in yellow, the downwards oscillation with period 2 (DO2) in green and finally the downwards oscillation with period 4 (DO4) in orange.

The basins of attraction corresponding to the values $\gamma=0.2,0.23$ and 0.2725 are shown in Figure 13. The relative areas of the basins of attraction for $\gamma \in[0.05,0.6]$ are listed in Table 11. Again the positive and negative rotations have been listed together as any difference in the size of their basins of attraction is expected to be due to numerical inaccuracies.

In Figure 14, there is a large jump in the relative area of the basin of attraction for the upwards fixed point $(\mathrm{FP})$ between the values of $\gamma=0.22$ and 0.225 , approximately: this is due to the appearance of the oscillatory solution which oscillates about the downwards pointing fixed point $(\xi=\pi)$. For values of $\gamma$ slightly larger than 0.22 large amounts of phase space move close to the solution DO2, where they remain for long periods of time; however they do not land on the solution 
and are eventually attracted to FP. The percentage of phase space which does this is marked in Figure 14 by a dotted line, which becomes solid when the trajectories remain on the solution for all time (however, see comments below).

\begin{tabular}{|c|c|c|c|c|c|c|c|}
\hline & \multicolumn{6}{|c|}{ Basin of Attraction \% } \\
\hline & & $\mathrm{FP}$ & DFP & $\mathrm{PR} / \mathrm{NR}$ & PR2/NR2 & DO2 & $\mathrm{DO} 4$ \\
\hline \multirow{22}{*}{$\gamma$} & 0.0500 & 4.30 & 0.00 & 0.00 & 47.85 & 0.00 & 0.00 \\
\hline & 0.0750 & 5.08 & 0.00 & 0.00 & 47.46 & 0.00 & 0.00 \\
\hline & 0.0900 & 7.41 & 0.00 & 0.00 & 46.30 & 0.00 & 0.00 \\
\hline & 0.1000 & 8.51 & 0.00 & 45.74 & 0.00 & 0.00 & 0.00 \\
\hline & 0.1700 & 49.65 & 0.00 & 25.17 & 0.00 & 0.00 & 0.00 \\
\hline & 0.2000 & 64.31 & 0.00 & 17.84 & 0.00 & 0.00 & 0.00 \\
\hline & 0.2230 & 72.09 & 0.00 & 13.95 & 0.00 & 0.00 & 0.00 \\
\hline & 0.2250 & 27.60 & 0.00 & 13.59 & 0.00 & 45.22 & 0.00 \\
\hline & 0.2300 & 25.00 & 0.00 & 12.68 & 0.00 & 49.61 & 0.00 \\
\hline & 0.2500 & 15.87 & 0.00 & 8.49 & 0.00 & 67.16 & 0.00 \\
\hline & 0.2690 & 16.50 & 0.00 & 2.13 & 0.00 & 79.25 & 0.00 \\
\hline & 0.2694 & 17.26 & 0.00 & 0.00 & 0.00 & 82.74 & 0.00 \\
\hline & 0.2700 & 17.28 & 0.00 & 0.00 & 0.00 & 82.73 & 0.00 \\
\hline & 0.2725 & 17.21 & 0.00 & 0.00 & 0.00 & 79.44 & 3.35 \\
\hline & 0.2800 & 17.30 & 0.00 & 0.00 & 0.00 & 82.70 & 0.00 \\
\hline & 0.2900 & 17.30 & 0.00 & 0.00 & 0.00 & 82.70 & 0.00 \\
\hline & 0.3000 & 16.97 & 0.00 & 0.00 & 0.00 & 83.03 & 0.00 \\
\hline & 0.4600 & 9.61 & 0.00 & 0.00 & 0.00 & 90.39 & 0.00 \\
\hline & 0.4700 & 9.80 & 90.20 & 0.00 & 0.00 & 0.00 & 0.00 \\
\hline & 0.5000 & 10.06 & 89.94 & 0.00 & 0.00 & 0.00 & 0.00 \\
\hline & 0.5500 & 10.32 & 89.68 & 0.00 & 0.00 & 0.00 & 0.00 \\
\hline & 0.6000 & 8.79 & 91.21 & 0.00 & 0.00 & 0.00 & 0.00 \\
\hline
\end{tabular}

Table 11: Relative areas of the basins of attraction with constant damping coefficient $\gamma$. The solutions are named as per Figures 12 and 13 with the addition of the downwards fixed point (DFP) and the rotating period 2 solutions (PR2/NR2). For details on the DO2 solution we refer to the text.

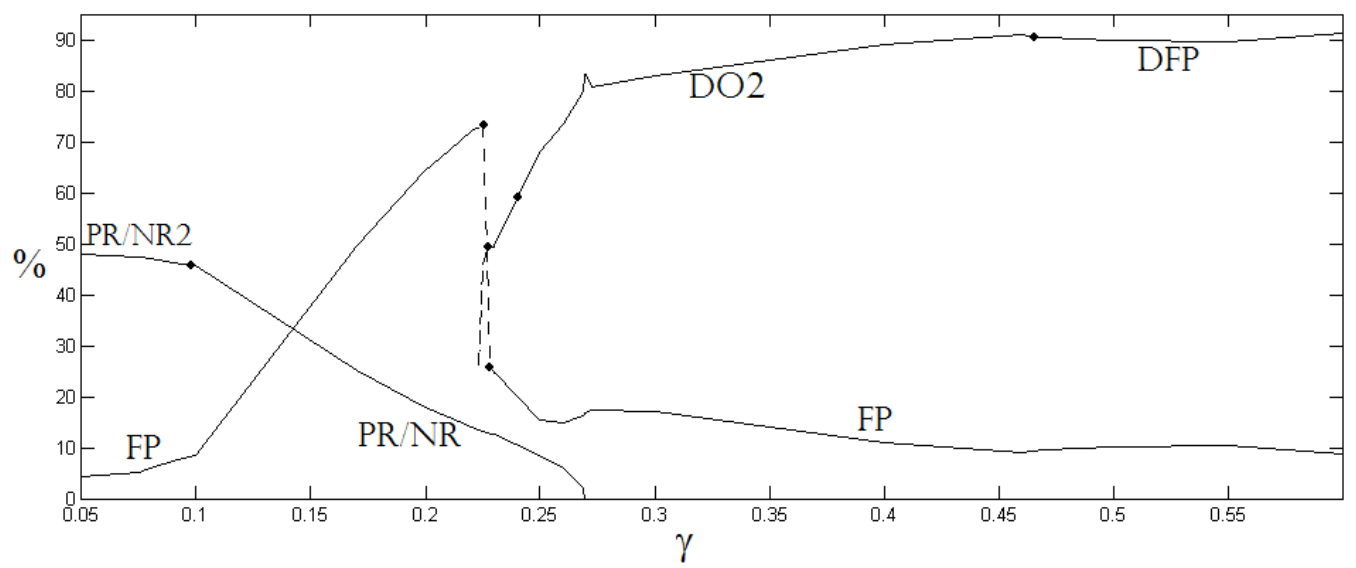

Figure 14: Relative sizes of basins of attraction with constant $\gamma$ as per Table 11. The lines are labeled as in Table 11 and regions in which a bifurcation takes place are marked with a dot. The basin of attraction for the oscillatory solution with period 4 (DO4), has not been included due to its small size and the solutions low range of persistence with respect to $\gamma$. The broken lines for FP and DO2 represent areas of transition just before DO2 (and the solutions created by the period doubling bifurcation) becomes stable, see text. 
The solution DO4 listed in Table 11 is found to persist only in the interval [0.272, 0.27422], where it only attracts a small amount of the phase space. As such it has not been included in Figure 14

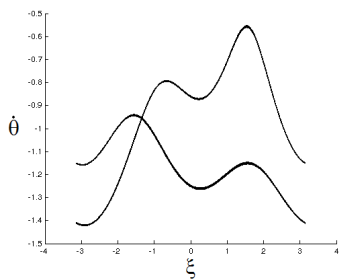

(a)

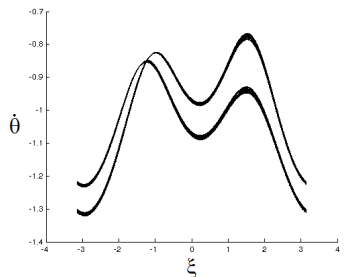

(b)

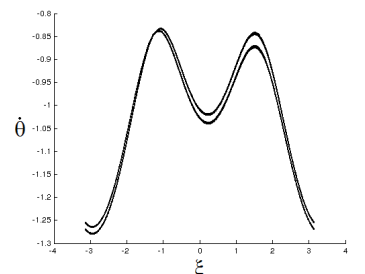

(c)

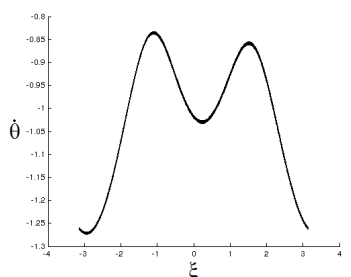

(d)

Figure 15: The transition from the period 2 rotating solutions to the period 1 rotating solutions. $\gamma=0.09$, $0.094,0.096$ and 0.098 from (a) to (d) respectively.

Numerical simulations can be used to find estimates for the values of $\gamma$ at which solutions appear/disappear. This is done by starting with initial conditions on a solution, then allowing the parameter $\gamma$ to be varied to see for which value that solution vanishes. In Figure 15 the transition from the period 2 rotating solution to the period 1 rotating solution can be observed: by moving towards smaller values of $\gamma$, this corresponds to a period doubling bifurcation [21, 13] (period halving, if we think of $\gamma$ as increasing). Similarly, starting on the period 1 rotating attractor and increasing further $\gamma$, the solution disappears at $\gamma \approx 0.2694$.

The same analysis can be done for the oscillatory solutions. We find that the downwards oscillatory solution labeled DO2 persists for $\gamma$ in the interval $[0.224,0.46]$, approximately. However such a solution is really a period 2 solution only for $\gamma$ greater than $\gamma \approx 0.24$. In the interval $[0.224,0.24]$ the trajectory is "thick", see Figure 16. only due to its similarity to the solution DO2 and to prevent Table 11 having yet more columns, the basin of attraction of these solutions in that range has also been listed under that of DO2. Nevertheless, by moving $\gamma$ backwards starting from 0.24 we have a sequence of period doubling bifurcations, corresponding to values of $\gamma$ closer and closer to each other. A period doubling cascade is expected to lead to a chaotic attractor, which, however, may survive only for a tiny window of values of $\gamma$ (at $\gamma=0.223$ it has already definitely disappeared) and has a very small basin of attraction (for $\gamma$ getting closer to the value 0.223 its relative area goes to zero). The appearance of chaotic attractors for small sets of parameters and with small basins of attraction has been observed in similar contexts of multistable dissipative systems close to the conservative limit [15. For the value $\gamma=0.223$ numerical simulations find that trajectories remain in the region of phase space occupied by DO2 for a long time, before eventually moving onto the fixed point. As $\gamma$ increases further towards $\gamma \approx 0.46$, the amplitude of the period 2 oscillatory solution gradually decreases and taking $\gamma$ larger causes a slow spiral into the downwards fixed point, which now becomes stable.

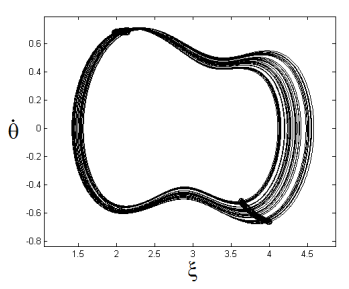

(a)

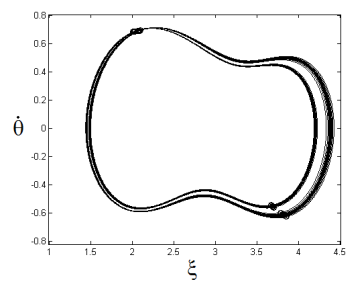

(b)

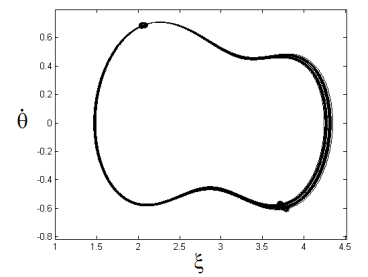

(c)

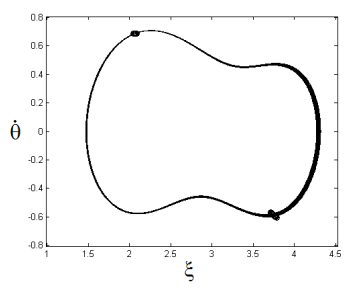

(d)

Figure 16: The solution DO2 for different values of time independent $\gamma$. As the damping coefficient is increased, the solution becomes more clearly defined: this is due to a period halving bifurcation which stops when the period becomes 2. The damping coefficient is $\gamma=0.23,0.235,0.239$ and 0.24 from (a) to (d), respectively. The position of the trajectory at every $2 \pi$, i.e the period of the forcing, is shown by circles. 


\subsection{Increasing dissipation}

As mentioned in Section 3.1. as $\gamma$ increases from $\gamma_{0}$ to $\gamma_{1}$ it is expected that taking $T_{0}$ larger causes the sizes of the basins of attraction to tend towards the sizes of the corresponding basins when $\gamma=\gamma_{0}$, when the set of attractors remains the same for all values in between. If an attractor is replaced by a new attractor (by bifurcation), then the new attractor inherits the basin of attraction of the old one.

We shall begin by fixing $\gamma_{1}=0.2$ and $\gamma_{0} \in[0.05,0.2]$, as for $\gamma=\gamma_{1}$ the basins of attraction are not so sensitive to initial conditions, see Figure 13(a), and for $\gamma$ in that range the set of attractors consists only of the the upwards fixed point and two rotating solutions; moreover the profiles of the corresponding relative areas plotted in Figure 14 are rather smooth and do not present any sharp jumps.

\begin{tabular}{|c|c|c|c|}
\cline { 3 - 4 } \multicolumn{2}{c|}{} & \multicolumn{2}{c|}{ Basin of Attraction \% } \\
\cline { 3 - 4 } \multicolumn{1}{c|}{} & 0 & FP & PR/NR \\
\cline { 2 - 4 } & 25 & 51.31 & 17.84 \\
\cline { 2 - 4 }$T_{0}$ & 50 & 32.09 & 24.30 \\
\cline { 2 - 4 } & 75 & 23.48 & 33.95 \\
\cline { 2 - 4 } & 100 & 17.09 & 48.26 \\
\cline { 2 - 4 } & 200 & 6.85 & 46.45 \\
\cline { 2 - 4 } & 500 & 4.35 & 47.82 \\
\hline & 1000 & 4.15 & 47.92 \\
\cline { 2 - 4 } & 1500 & 4.09 & 47.96 \\
\hline
\end{tabular}

Table 12: Relative areas of the basins of attraction with $\gamma_{0}=0.05, \gamma_{1}=0.2$ and $T_{0}$ varying.

\begin{tabular}{|c|c|c|c|}
\cline { 3 - 4 } \multicolumn{2}{c|}{} & \multicolumn{2}{c|}{ Basin of Attraction \% } \\
\cline { 2 - 4 } \multicolumn{1}{c|}{} & FP & PR/NR \\
\hline \multirow{5}{*}{$T_{0}$} & 0 & 64.31 & 17.84 \\
\cline { 2 - 4 } & 25 & 49.06 & 25.47 \\
\cline { 2 - 4 } & 50 & 38.54 & 30.73 \\
\cline { 2 - 4 } & 75 & 31.25 & 34.37 \\
\cline { 2 - 4 } & 100 & 25.67 & 37.16 \\
\cline { 2 - 4 } & 150 & 18.12 & 40.94 \\
\cline { 2 - 4 } & 200 & 14.14 & 42.93 \\
\cline { 2 - 4 } & 500 & 9.81 & 45.09 \\
\cline { 2 - 4 } & 1000 & 9.45 & 45.27 \\
\hline
\end{tabular}

Table 13: Relative areas of the basins of attraction with $\gamma_{0}=0.1, \gamma_{1}=0.2$ and $T_{0}$ varying.

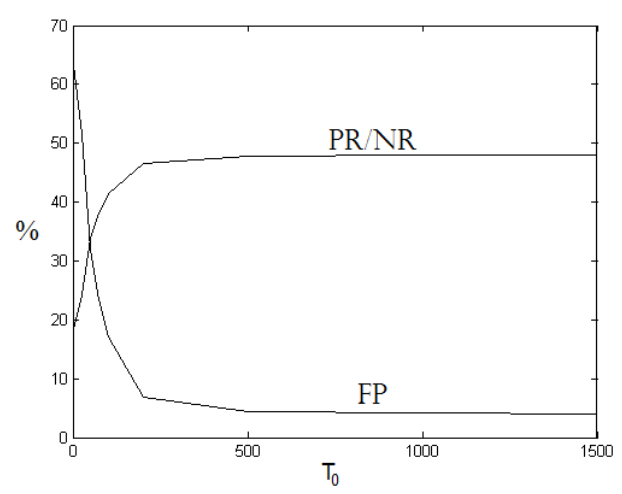

Figure 17: Plot of the relative areas of the basins of attraction as per Table 12 .

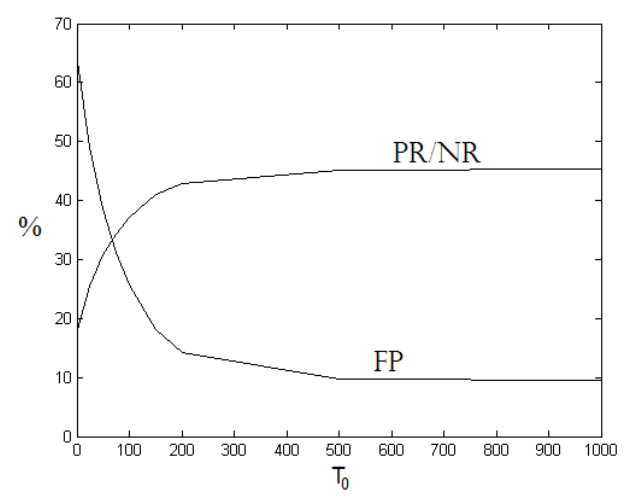

Figure 18: Plot of the relative areas of the basins of attraction as per Table 13 .

Tables 12,13 and 14 show the relative area of each basin of attraction as $\gamma$ increases from 0.05 , 0.1 and 0.17 , respectively, to 0.2 with varying $T_{0}$. It can be seen from the results in Tables 13 to 14 that the numerical simulations are in agreement with the above expectation. With the exception of Table 12 the relative areas of the basins of attraction tend towards those when $\gamma$ is kept constant at $\gamma_{0}$. The exception of the case of Table 12 is due to the fact that the set of attractors has changed as $\gamma$ passes from 0.05 to 0.2 : the period 2 rotating solutions have been destroyed and replaced by the period 1 rotating solutions. However, when the transition occurs, the new attractors are located in 
phase space very close to the previous ones and we find that the initial conditions which were heading towards or had indeed landed on the period 2 rotating solutions move onto the now present period 1 rotating solutions. On the other hand, when the damping coefficient crosses the value $\gamma \approx 0.1$, the attractor undergoes topological changes, but, apart from that, the transition is rather smooth: the location in phase space and the basin of attraction change continuously. In conclusion, we find that the relative areas of the basins of attraction for the two period 1 rotating attractors $(\mathrm{PR} / \mathrm{NR})$ tend towards those the now destroyed period 2 rotating attractors (PR2/NR2) had at $\gamma=\gamma_{0}$. As in Section 3 we expect that the sizes of the basin of attraction at $\gamma=\gamma_{0}$ are recovered asymptotically as $T_{0} \rightarrow \infty$. Nevertheless, once more, the larger $T_{0}$ the smaller is the variation in the relative area: for instance in Table 12 for $T_{0}=100$ the relative area of the basin of attraction of the fixed point has become nearly $1 / 4$ of the value for $\gamma=0.2$ constant, while in order to have a further reduction by a factor of 4 one has to take $T_{0}=1000$.

\begin{tabular}{|c|c|c|c|}
\cline { 3 - 4 } \multicolumn{2}{c|}{} & \multicolumn{2}{c|}{ Basin of Attraction \% } \\
\cline { 2 - 4 } \multicolumn{1}{c|}{} & FP & PR/NR \\
\hline \multirow{5}{*}{$T_{0}$} & 0 & 64.31 & 17.84 \\
\cline { 2 - 4 } & 25 & 58.54 & 20.73 \\
\cline { 2 - 4 } & 50 & 56.78 & 21.62 \\
\cline { 2 - 4 } & 100 & 55.41 & 22.29 \\
\cline { 2 - 4 } & 200 & 53.69 & 23.15 \\
\cline { 2 - 4 } & 500 & 51.93 & 24.04 \\
\cline { 2 - 4 } & 1000 & 50.80 & 24.60 \\
\cline { 2 - 4 } & 1500 & 50.62 & 24.69 \\
\hline
\end{tabular}

Table 14: Relative areas of the basins of attraction with $\gamma_{0}=0.17, \gamma_{1}=0.2$ and $T_{0}$ varying.

\begin{tabular}{|c|c|c|c|c|}
\hline & \multicolumn{3}{|c|}{ Basin of Attraction \% } \\
\hline & & $\mathrm{FP}$ & $\mathrm{PR} / \mathrm{NR}$ & $\mathrm{DO} 2$ \\
\hline \multirow{16}{*}{$T_{0}$} & 0 & 25.00 & 12.68 & 49.61 \\
\hline & 10 & 24.86 & 13.81 & 47.52 \\
\hline & 15 & 24.34 & 14.84 & 45.98 \\
\hline & 20 & 24.43 & 15.57 & 44.43 \\
\hline & 25 & 24.77 & 16.04 & 43.15 \\
\hline & 50 & 27.60 & 16.98 & 38.45 \\
\hline & 75 & 30.12 & 17.28 & 35.32 \\
\hline & 100 & 33.08 & 17.42 & 32.08 \\
\hline & 150 & 38.29 & 17.56 & 26.58 \\
\hline & 200 & 42.60 & 17.66 & 22.08 \\
\hline & 300 & 49.36 & 17.73 & 15.18 \\
\hline & 400 & 54.20 & 17.75 & 10.30 \\
\hline & 500 & 57.37 & 17.77 & 7.08 \\
\hline & 1000 & 63.39 & 17.78 & 1.06 \\
\hline & 1500 & 64.26 & 17.79 & 0.16 \\
\hline & 2000 & 64.38 & 17.80 & 0.03 \\
\hline
\end{tabular}

Table 15: Relative areas of the basins of attraction with $\gamma_{0}=0.2, \gamma_{1}=0.23$ and $T_{0}$ varying.
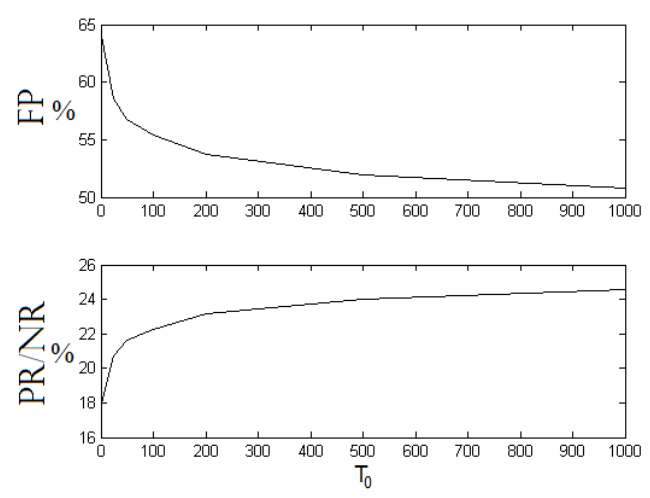

Figure 19: Plot of the relative areas of the basins of attraction as per Table 14 .

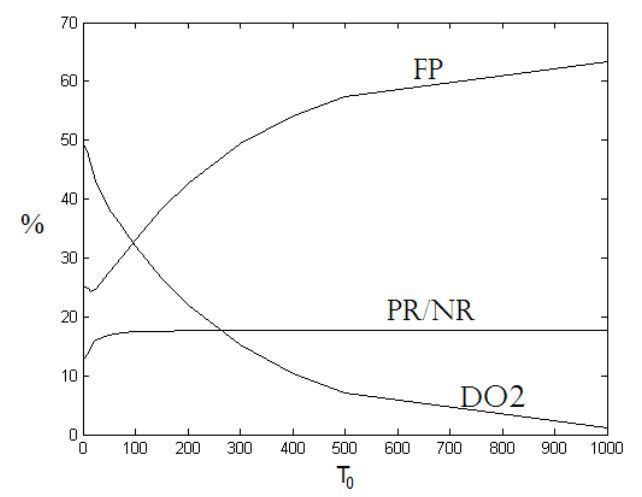

Figure 20: Plot of the relative areas of the basins of attraction as per Table 15 
We now consider the case where either $\gamma_{0}=0.2$ and $\gamma_{1}=0.23$ or 0.2725 or $\gamma_{0}=0.23$ and $\gamma_{1}=0.2725$. Such values for $\gamma$ offer more complexities as not only are there more attractors to consider, but one may have attractors (PR and NR) that are destroyed without leaving any trace. When this happens it is not obvious which persisting attractor will inherit their basins of attraction. The result of this could cause the final basins of attraction to be drastically different from those for constant $\gamma$ and even not monotonically increasing or decreasing as the value of $T_{0}$ is increased.

In Table 15 we see that initially, for values of $T_{0}$ not too large, the basin of attraction of FP slightly reduces in size, while those of the rotating solutions PR/NR increase substantially. Instead, for larger values of $T_{0}$, the basin of attraction of FP increases appreciably, while those of PR/NR increase very slowly. Apparently, the rotating solutions react more quickly as $\gamma$ is varied, attracting phase space faster, so that the relative areas of their basins of attraction tend towards the values at $\gamma=\gamma_{0}$ for shorter initial times $T_{0}$. It would be interesting to study further this phenomenon.

When $\gamma(t)$ varies from $\gamma=0.2$ to $\gamma=0.2725$ and from $\gamma=0.23$ to $\gamma=0.2725$, the rotating solutions $\mathrm{PR} / \mathrm{NR}$ disappear, so that their basins of attractions are absorbed by the persisting attractors. In Table 16 one sees a very slow movement towards global attraction of the upwards fixed point, which is the only attractor persisting for both $\gamma_{0}$ and $\gamma_{1}$. However even taking $T_{0}=5000$ is not enough for the asymptotic behaviour to be approached. The results in Table 17] show that, by taking $T_{0}$ larger and larger, the relative areas of the basins of attraction of $\mathrm{FP}$ and DO4 both tend to the values corresponding to $\gamma_{0}=0.23$ (in particular the basin of attraction of DO 4 becomes negligible). Nearly all trajectories which were converging towards the rotating solutions before the latter disappeared are attracted by the period two oscillations. This could be due to the fact that DO2 is the closest attractor in phase space which persists at both $\gamma_{0}$ and $\gamma_{1}$.

\begin{tabular}{|c|c|c|c|c|}
\hline & \multicolumn{3}{|c|}{ Basin of Attraction \% } \\
\hline & & $\mathrm{FP}$ & DO2 & DO4 \\
\hline \multirow{23}{*}{$T_{0}$} & 0 & 17.21 & 79.44 & 3.35 \\
\hline & 25 & 18.63 & 78.33 & 3.04 \\
\hline & 50 & 20.32 & 79.29 & 0.39 \\
\hline & 75 & 23.38 & 76.52 & 0.12 \\
\hline & 100 & 25.71 & 74.27 & 0.02 \\
\hline & 150 & 28.45 & 71.55 & 0.01 \\
\hline & 200 & 30.92 & 69.08 & 0.00 \\
\hline & 300 & 35.70 & 64.30 & 0.00 \\
\hline & 400 & 39.82 & 60.18 & 0.00 \\
\hline & 500 & 42.76 & 57.24 & 0.00 \\
\hline & 980 & 54.19 & 45.81 & 0.00 \\
\hline & 990 & 54.39 & 45.81 & 0.00 \\
\hline & 995 & 54.30 & 45.70 & 0.00 \\
\hline & 1000 & 90.11 & 9.89 & 0.00 \\
\hline & 1005 & 54.58 & 45.43 & 0.00 \\
\hline & 1010 & 54.52 & 45.48 & 0.00 \\
\hline & 1020 & 90.34 & 9.66 & 0.00 \\
\hline & 1030 & 54.81 & 45.19 & 0.00 \\
\hline & 1050 & 55.13 & 44.87 & 0.00 \\
\hline & 1500 & 59.78 & 40.22 & 0.00 \\
\hline & 2000 & 62.12 & 37.88 & 0.00 \\
\hline & 3000 & 63.89 & 36.11 & 0.00 \\
\hline & 5000 & 64.29 & 35.71 & 0.00 \\
\hline
\end{tabular}

Table 16: Relative areas of the basins of attraction with $\gamma_{0}=0.2, \gamma_{1}=0.2725$ and $T_{0}$ varying.
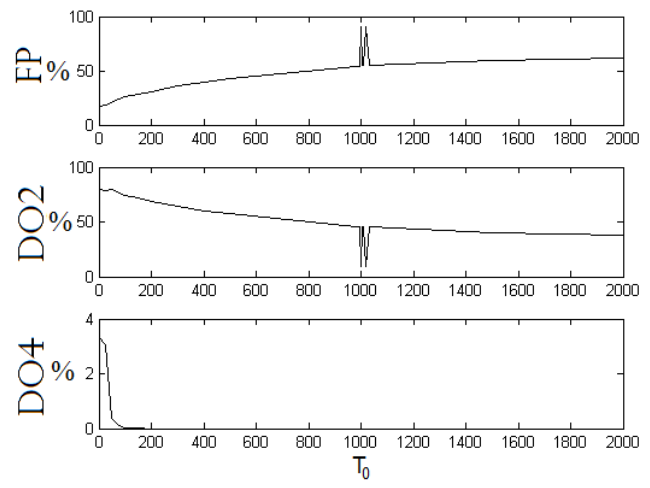

Figure 21: Plot of the relative areas of the basins of attraction as per Table 16 .

However, the more striking feature of Figures 21 and 22 are the jumps corresponding $T_{0}=1000$ 
in the prior and $T_{0}=100$ and $T_{0}=500$ in the latter. Moreover such jumps are very localised: for instance in Figure 22 for $T_{0}=99$ and $T_{0}=100$ the basins of attraction of FP and DO2 are found to be about $44 \%$ and $56 \%$, respectively, whereas by slightly increasing or decreasing $T_{0}$ they settle around $20 \%$ and $80 \%$. The quantity of phase space exchanged in these instances is roughly equal to that attracted to the rotating solutions for $\gamma_{0}$. For particular values of $T_{0}$ when the rotating attractors disappear their trajectories move to the upwards fixed point rather than the period 2 oscillations. The reason for this to happen is not clear. Moreover, note that in principle there could be other jumps, corresponding to values of $T_{0}$ which have not been investigated: however, it seems hard to make any prediction as far as it remains unclear how the disappearing basins of attractions are absorbed by the persisting ones.

\begin{tabular}{|c|c|c|c|c|}
\hline & \multicolumn{3}{|c|}{ Basin of Attraction \% } \\
\hline & & $\mathrm{FP}$ & $\mathrm{DO} 2$ & DO4 \\
\hline \multirow{22}{*}{$T_{0}$} & 0 & 17.21 & 79.44 & 3.35 \\
\hline & 25 & 17.96 & 79.45 & 2.60 \\
\hline & 50 & 16.04 & 83.36 & 0.60 \\
\hline & 75 & 18.39 & 80.27 & 1.34 \\
\hline & 90 & 19.56 & 80.38 & 0.05 \\
\hline & 95 & 20.05 & 79.91 & 0.04 \\
\hline & 97 & 20.08 & 79.89 & 0.04 \\
\hline & 98 & 20.01 & 79.96 & 0.03 \\
\hline & 99 & 44.14 & 55.83 & 0.03 \\
\hline & 100 & 43.96 & 56.01 & 0.03 \\
\hline & 101 & 20.54 & 79.42 & 0.03 \\
\hline & 105 & 20.40 & 79.57 & 0.03 \\
\hline & 110 & 20.79 & 79.19 & 0.02 \\
\hline & 125 & 21.55 & 78.45 & 0.01 \\
\hline & 150 & 22.46 & 77.54 & 0.00 \\
\hline & 200 & 23.33 & 76.67 & 0.00 \\
\hline & 300 & 24.06 & 75.94 & 0.00 \\
\hline & 400 & 24.36 & 75.64 & 0.00 \\
\hline & 490 & 24.54 & 75.46 & 0.00 \\
\hline & 500 & 49.45 & 50.55 & 0.00 \\
\hline & 510 & 24.42 & 75.58 & 0.00 \\
\hline & 1000 & 24.81 & 75.19 & 0.00 \\
\hline
\end{tabular}

Table 17: Relative areas of the basins of attraction with $\gamma_{0}=0.23, \gamma_{1}=0.2725$ and $T_{0}$ varying.
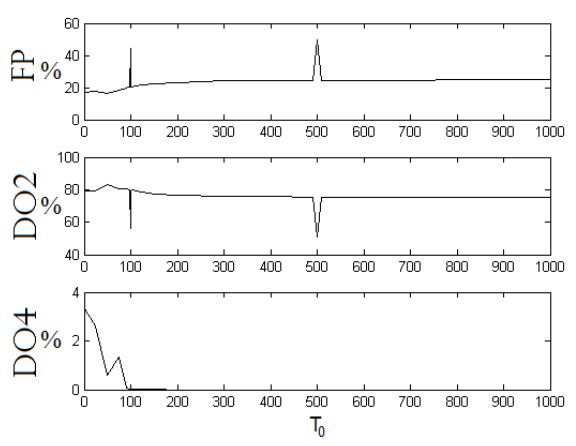

Figure 22: Plot of the relative areas of the basins of attraction as per Table 17.

\subsection{Decreasing dissipation}

Tables 18, 19, 20 and 21 and the corresponding Figures 23, 24, 25, and 26 illustrate the cases when dissipation decreases over an initial period of time $T_{0}$. We have considered the cases with $\gamma_{0}=0.23$, 0.02725 and $\gamma_{1}=0.2$, with $\gamma_{0}=0.2725$ and $\gamma_{1}=0.23$ and with $\gamma_{0}=0.3$ and $\gamma_{1}=0.2725$.

In particular they show that if the set of attractors at $\gamma=\gamma_{1}$ is a proper subset of the set of attractors which exist at $\gamma=\gamma_{0}$, then, as $T_{0} \rightarrow \infty$, the basin of attraction of each attractor which exists at $\gamma_{1}$ turns out to have a relative area which tend to be greater than or equal to that found for $\gamma=\gamma_{0}$. In Table 18 we consider the situation in which the attractor DO2, which has a large basin of attraction for $\gamma_{0}=0.23$, is no longer present when $\gamma(t)$ has reached the final value $\gamma_{1}=0.2$ : as a consequence the trajectories which would be attracted by DO2 at $\gamma=\gamma_{0}$ end up onto the other attractors: in fact most of them are attracted by the fixed point. 


\begin{tabular}{|c|c|c|c|}
\cline { 3 - 4 } \multicolumn{2}{c|}{} & \multicolumn{2}{c|}{ Basin of Attraction \% } \\
\cline { 3 - 4 } \multicolumn{2}{c|}{} & FP & PR/NR \\
\hline \multirow{4}{*}{$T_{0}$} & 0 & 64.31 & 17.84 \\
\cline { 2 - 4 } & 25 & 70.69 & 14.65 \\
\cline { 2 - 4 } & 50 & 72.57 & 13.72 \\
\cline { 2 - 4 } & 75 & 73.24 & 13.38 \\
\cline { 2 - 4 } & 100 & 73.57 & 13.22 \\
\cline { 2 - 4 } & 200 & 74.10 & 12.95 \\
\cline { 2 - 4 } & 500 & 74.42 & 12.79 \\
\hline
\end{tabular}

Table 18: Relative areas of the basins of attraction with $\gamma_{0}=0.23, \gamma_{1}=0.2$ and $T_{0}$ varying.

\begin{tabular}{|c|c|c|c|}
\hline & \multicolumn{2}{|c|}{ Basin of Attraction \% } \\
\hline & & $\mathrm{FP}$ & $\mathrm{PR} / \mathrm{NR}$ \\
\hline \multirow{13}{*}{$T_{0}$} & 0 & 64.31 & 17.84 \\
\hline & 5 & 65.80 & 17.10 \\
\hline & 10 & 69.52 & 15.24 \\
\hline & 15 & 74.40 & 12.80 \\
\hline & 20 & 77.90 & 11.05 \\
\hline & 25 & 80.38 & 9.81 \\
\hline & 50 & 86.22 & 6.89 \\
\hline & 75 & 88.64 & 5.68 \\
\hline & 100 & 90.07 & 4.97 \\
\hline & 200 & 92.79 & 3.61 \\
\hline & 500 & 95.92 & 2.04 \\
\hline & 1000 & 99.34 & 0.33 \\
\hline & 1500 & 99.35 & 0.32 \\
\hline
\end{tabular}

Table 19: Relative areas of the basins of attraction with $\gamma_{0}=0.2725, \gamma_{1}=0.2$ and $T_{0}$ varying.

\begin{tabular}{|c|c|c|c|c|}
\hline & \multicolumn{3}{|c|}{ Basin of Attraction \% } \\
\hline & & FP & $\mathrm{PR} / \mathrm{NR}$ & DO2 \\
\hline \multirow{7}{*}{$T_{0}$} & 0 & 25.00 & 12.68 & 49.61 \\
\hline & 25 & 24.73 & 7.44 & 60.40 \\
\hline & 50 & 19.67 & 5.35 & 69.63 \\
\hline & 75 & 17.13 & 4.44 & 73.99 \\
\hline & 100 & 16.42 & 3.88 & 75.83 \\
\hline & 200 & 16.29 & 2.72 & 78.28 \\
\hline & 500 & 17.03 & 0.76 & 81.45 \\
\hline
\end{tabular}

Table 20: Relative areas of the basins of attraction with $\gamma_{0}=0.2725, \gamma_{1}=0.23$ and $T_{0}$ varying.
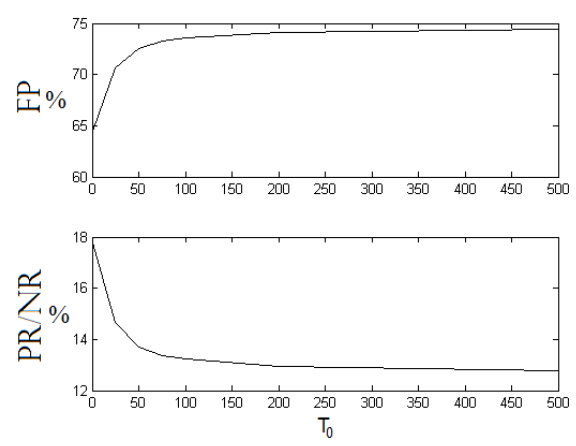

Figure 23: Plot of the relative areas of the basins of attraction as per Table 18
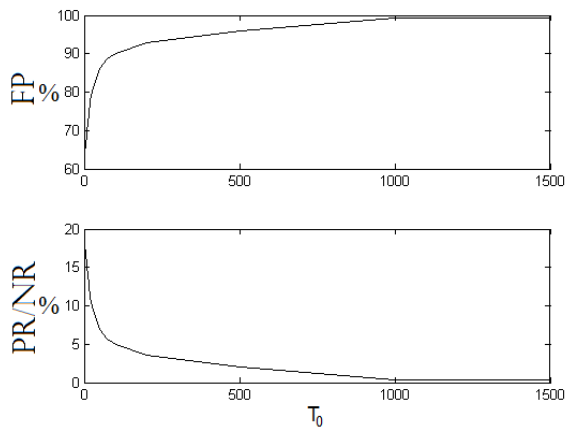

Figure 24: Plot of the relative areas of the basins of attraction as per Table 19 .

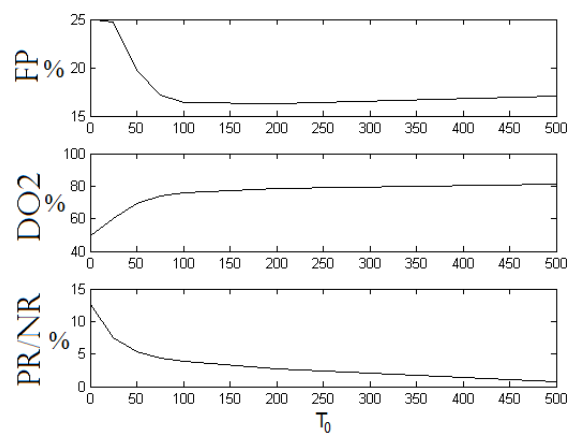

Figure 25: Plot of the relative areas of the basins of attraction as per Table 20

We also notice the interesting features in Table 19 as the fixed point is the only attractor which 
exists for both $\gamma_{0}$ and $\gamma_{1}$, we find that as $T_{0}$ increases its basin of attraction tends towards $100 \%$, which corresponds to attraction of the entire phase space, up to a zero-measure set. This happens despite the fact that $\gamma(t)$ does not pass through any value for which global attraction to the fixed point is satisfied. It also suggests that it is possible to provide conditions on the intersection of the two sets $\mathcal{A}_{0}$ and $\mathcal{A}_{1}$ of the attractors corresponding to $\gamma_{0}$ and $\gamma_{1}$, respectively, in order to obtain that all trajectories move towards the same attractor when the time $T_{0}$ over which $\gamma(t)$ is varied is sufficiently large. In particular, it is remarkable that it is possible to create an attractor for almost all trajectories by suitably tuning the damping coefficient as a function of time.

In Table 20, the relative areas of the rotating solutions, which are absent at $\gamma=\gamma_{0}$, tend to become negligible when $T_{0}$ is large. Similarly, in Table 21, the basin of attraction of the period 4 oscillating attractor, which exists only for the final value $\gamma_{1}$ of the damping coefficient, tends to disappear when $T_{0}$ is taken large enough. This confirms the general expectation: the basin of attraction of the disappearing attractor is absorbed by the closer attractor, that is the solution DO2 in this case.

\begin{tabular}{|c|c|c|c|c|}
\cline { 3 - 5 } \multicolumn{2}{c|}{} & \multicolumn{3}{c|}{ Basin of Attraction \% } \\
\cline { 3 - 5 } \multicolumn{2}{c|}{} & FP & DO2 & DO4 \\
\hline \multirow{4}{*}{$T_{0}$} & 0 & 17.21 & 79.44 & 3.35 \\
\hline & 25 & 17.15 & 80.99 & 1.86 \\
\cline { 2 - 5 } & 50 & 16.83 & 83.03 & 0.14 \\
\cline { 2 - 5 } & 75 & 16.75 & 83.24 & 0.01 \\
\cline { 2 - 5 } & 100 & 16.79 & 83.21 & 0.00 \\
\cline { 2 - 5 } & 200 & 16.81 & 83.19 & 0.00 \\
\cline { 2 - 5 } & 500 & 16.80 & 83.20 & 0.00 \\
\hline
\end{tabular}

Table 21: Relative areas of the basins of attraction with $\gamma_{0}=0.3, \gamma_{1}=0.2725$ and $T_{0}$ varying.
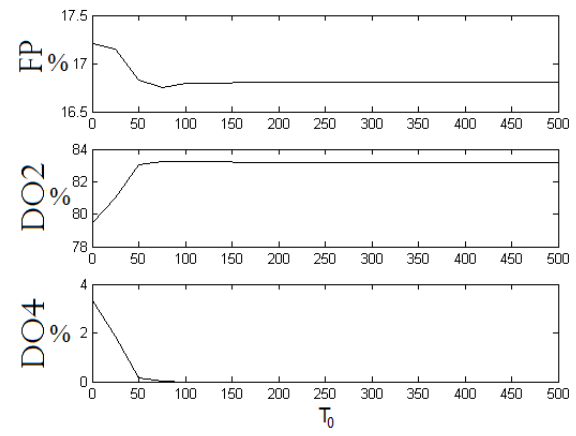

Figure 26: Plot of the relative areas of the basins of attraction as per Table 21

\section{$5 \quad$ Numerical Methods}

The two main numerical methods implemented for the simulations throughout were a variable order Adams-Bashforth-Moulton method and the method of analytic continuation [10, 23, 12, 17, 34, 36]; the latter consists in a numerical implementation of the Frobenius method. Also used to check the results was a Runge-Kutta method. The Adams-Bashforth-Moulton integration scheme used is the built in integrator found in MATLAB, ODE113, whereas the programs based on the method of analytic continuation and Runge-Kutta scheme were written in C. Of the three methods, the slowest was the Adams-Bashforth-Moulton method, however it was found that the method worked well for the system with the chosen parameter values and the results produced were reliable.

Both the Adams-Bashforth-Moulton and Runge-Kutta methods are standard methods for solving ODEs of this type. When implementing these two integrators to calculate the basins of attraction, two different methods for both choosing initial conditions in phase space and classifying attractors were used. The first method for picking initial conditions was to take a mesh of equally spaced points in the phase space: this method ensures uniform coverage of the phase space. When taking this approach a mesh of either 321141 or 503289 points was used depending on the desired accuracy. The second method was to take random initial conditions: this can be done by choosing initial conditions from a stream of random points, which allows the user to use the same random initial conditions in each simulation if required. This method is often preferred as the accuracy of the estimates of the relative areas of the basins of attraction compared to the number of initial conditions used can be calculated 29]; also it is easier to run additional simulations for extra random points to improve estimates later on, when needed. When using random points, the number of points used to calculate the basins of attraction was 300000 or 400000 depending on the expected complexity 
of the system under given parameters. In some cases where extra accuracy was required due to some attractors having particularly small basins of attraction, additional 200000 or 300000 random points were used. This allowed us to obtain an error less than 0.20 on the relative areas of the basins of attraction. In the most delicate cases, where more precise estimates were needed to distinguish between values very close to each other (for instance in Table 4), the error was made smaller by increasing the number of points. We decided to express in all cases the relative areas up to the second decimal digit because often further increasing the number of points did not alter appreciably that digit.

To detect and classify solutions, two methods can be used. The first method consists in finding all attractors as a first step, before computing the corresponding basins of attraction: this required a complete characterisation of both the period and the location in phase space of the attractors. In principle, this works very well, but has the downside of having to find initially all the attractors, and differentiate between those that occupy the same region in phase space. The second method for classifying the solutions was to create a library of solutions. This was created as the program ran and built up as new solutions were found. The solution of each integration was then checked against the library and, if not already known, was added. In this way, the program finds solutions as it goes, so has the advantage of the user not having to know the existing solutions in the system prior to calculating the basins of attraction.

The method of analytic continuation was implemented and produced results very similar to those of the Adams-Bashforth-Moulton method, but in general was much quicker to run. When implementing this method of integration, we only used random initial conditions in the phase space and the library method for classifying solutions. The reason for using the Adams-Bashforth-Moulton method, despite being the slowest of the three integrators, was for comparison with the method of analytic continuation. Analytic continuation has not previously been used for numerically integrating an ODE of the form (1.2), that is an equation with an infinite polynomial nonlinearity that satisfies an addition formula, thus it was good to have a reliable method to check results with. The method for using analytic continuation for integrating ODE's that have infinite polynomial nonlinearity that satisfy an addition formula will be described more extensively in [36]. The similarity in results of these completely different methods for integration, initial condition selection and solution classification provides reassurance and confidence that the results produced are accurate.

\section{Concluding remarks}

In this work we have numerically shown the importance of not only the final value of dissipation but its entire time evolution, for understanding the long time behaviour of the pendulum with oscillating support. This extends the work done in 3] to a system which, even for values of the parameters in the perturbation regime, exhibits richer and more varied dynamics, due to the presence of the separatrix in the phase space of the unperturbed system. In addition we have considered also values of the parameters beyond the perturbation regime (the inverted pendulum), where the system cannot be considered a perturbation of an integrable one. In particular this results in a more complicated scenario, with bifurcation phenomena and the appearance of attractors which exist only for values of the damping coefficient $\gamma$ in finite intervals away from zero, say $\gamma \in\left[\gamma_{1}, \gamma_{2}\right]$, with $\gamma_{1}>0$.

We have preliminarily studied the behaviour of the system in the case of constant dissipation. Firstly, in the perturbation regime, we have analytically computed to first order the threshold values below which the periodic attractors exist. We have also discussed why this approach fails due to intrinsic perturbation theory limitations, in particular why the method cannot be applied to stable cases of the upwards configuration or to solutions too close to the unperturbed separatrix. Next, we have studied numerically the dependence of the sizes of the basins of attraction on the damping coefficient.

Then we have explicitly considered the case of damping coefficient varying monotonically between two values and outlined a few expectations for the way in which the basins of attraction accordingly change with respect to the case of constant dissipation. These expectations were later illustrated and backed up with numerical simulations: in particular the relevance of the study of the dynamics 
at constant dissipation was argued at length. While the expectations account for many features observed numerically, there are still some facts which are difficult to explain, even at an heuristic level, and which would deserve further investigation, such as the relationship between the fixed point solution and the oscillating attractors, to better understand why in some cases they exchange large areas of their basins of attraction when the damping coefficient varies in time. More generally, an in-depth numerical study of the system with constant dissipation, also for other parameter values, would be worthwhile. In particular it would be interesting to perform a more detailed bifurcation analysis with respect to the parameter $\gamma$ and to study the system for very small values of the damping coefficient $\gamma$ (which relates to the spin-orbit model in celestial mechanics), both in the perturbation regime and for large values of the forcing amplitude. We think that, in order to study cases with very small dissipation, the method of analytical continuation briefly described in Section 5 could be particularly fruitful.

Some interesting features appeared in our analysis which would deserve further consideration are:

- the increase in the basin of attraction of the fixed point observed in Figure 3 when the damping coefficient becomes small enough;

- the appearance of the period 4 solution for a thin interval of values of the damping coefficient, as emerges in Table 11 .

- the rate at which the values of the relative areas of the basins attraction corresponding to the initial value $\gamma_{0}$ of $\gamma(t)$ are approached as the variation time $T_{0}$ increases;

- the way in which the basins of attraction of the disappearing attractors distribute among the persisting ones;

- the oscillations through which the relative areas of the basins of attraction approach the asymptotic value when taking larger and larger values of the variation time $T_{0}$, as observed for instance in Tables 16 and 17

- the jumps corresponding to $T_{0}=1000$ in Figure 21 and $T_{0}=100, T_{0}=500$ in Figure 22 ,

- the computation of the threshold values to second order, so as to include the rotating solutions found numerically in the perturbation regime investigated in Section 3

Finally, investigating analogous systems such as the pendulum with periodically varying length to see if similar dynamics occur would also be fascinating in its own right. Another interesting model to investigate further, especially in the case of very small dissipation, is the spin-orbit model already considered in 3, which is expected to be of relevance to understand the locking into the resonance $3: 2$ of the system Mercury-Sun.

\section{Acknowledgements}

The Adams-Bashforth-Moulton method used was MATLAB's ODE113. We thank Jonathan Deane for helpful conversations on analytic continuation and support with coding in C. This research was completed as part of an EPSRC funded PhD.

\section{A Global attraction to the two fixed points}

To compute the conditions for attraction to the origin we use the method outlined in 4]; see also [2. We define $f(\tau)$ as in $(1.3)$ and require $f(\tau)>0$ : the consequences of this restriction are that the method can only be applied to the downwards pointing pendulum when $\alpha>\beta$. Then we apply the Liouville transformation

$$
\tilde{\tau}=\int_{0}^{\tau} \sqrt{f(s)} \mathrm{d} s
$$


and write our equation 1.3 in terms of the new time $\tilde{\tau}$ as

$$
\theta_{\tilde{\tau} \tilde{\tau}}+\left(\frac{\tilde{f}(\tilde{\tau})_{\tilde{\tau}}}{2 \tilde{f}(\tilde{\tau})}+\frac{\gamma}{\sqrt{\tilde{f}(\tilde{\tau})}}\right) \theta_{\tilde{\tau}}+\sin \theta=0,
$$

where the subscript $\tilde{\tau}$ represents derivative with respect to the new time $\tilde{\tau}$ and $\tilde{f}(\tilde{\tau}):=f(\tau)$. This can be represented as the two-dimensional system on $\mathbb{T} \times \mathbb{R}$, by setting $x(\tilde{\tau})=\theta(\tilde{\tau})$ and writing

$$
x_{\tilde{\tau}}=y, \quad y_{\tilde{\tau}}=-\frac{y}{\sqrt{\tilde{f}}}\left(\frac{\tilde{f}_{\tilde{\tau}}}{2 \sqrt{\tilde{f}}}+\gamma\right)-\sin x,
$$

for which we have the energy $E(x, y)=1-\cos x+y^{2} / 2$. By setting $H(\tilde{\tau})=E(x(\tilde{\tau}), y(\tilde{\tau}))$, one finds

$$
H_{\tilde{\tau}}=-\frac{y^{2}}{\sqrt{\tilde{f}}}\left(\frac{\tilde{f}_{\tilde{\tau}}}{2 \sqrt{\tilde{f}}}+\gamma\right)
$$

thus $H_{\tilde{\tau}} \leq 0$, i.e $x, y$ are bounded given that $\gamma$ satisfies

$$
\gamma>-\min _{\tilde{\tau} \geq 0} \frac{\tilde{f}_{\tilde{\tau}}}{2 \sqrt{\tilde{f}}}=-\min _{\tau \geq 0} \frac{f^{\prime}}{2 f} .
$$

Moreover we have that for all $\tilde{\tau}>0$

$$
H(\tilde{\tau})+\int_{0}^{\tilde{\tau}} \frac{y^{2}}{\sqrt{\tilde{f}}}\left(\frac{\tilde{f}^{\prime}}{2 \sqrt{\tilde{f}}}+\gamma\right) \mathrm{d} s=H(0),
$$

so that, as $\tilde{\tau} \rightarrow \infty$, using the properties above we can arrive at

$$
\min _{s \geq 0}\left[\frac{1}{\sqrt{\tilde{f}}}\left(\frac{\tilde{f}_{\tilde{\tau}}}{2 \sqrt{\tilde{f}}}+\gamma\right)\right] \int_{0}^{\infty} y^{2}(s) \mathrm{d} s<\infty .
$$

Hence $y \rightarrow 0$ as time tends to infinity. There are two regions of phase space to consider. Any level curve of $H$ strictly inside the separatrix of the unperturbed pendulum is the boundary of a positively invariant set $D$ containing the origin: since $S=\left\{(x(\tilde{\tau}), y(\tilde{\tau})): H_{\tilde{\tau}}=0\right\} \cup D$ consists purely of the origin, we can apply the local Barbashin-Krasovsky-La Salle theorem [24] to conclude that every trajectory that begins strictly inside the separatrix will converge to the origin as $\tilde{\tau} \rightarrow+\infty$.

Outside of the separatrix we may use equation A.4, which shows the energy to be strictly decreasing while $y \neq 0$, provided $\gamma$ is chosen large enough, coupled with $y \rightarrow 0$ as time tends to infinity. The result is that all trajectories tend to the invariant points on the $x$-axis as time tends to infinity. One of two cases must occur: either the trajectory moves inside the separatrix or it does not. In the first instance we have already shown that the limiting solution is the origin. In the latter there is only one possibility. As all points on the $x$-axis are contained within the separatrix other than the unstable fixed point, the trajectory must move onto such a fixed point and hence belongs to its stable manifold, which is a zero-measure set. Therefore we conclude that a full measure set of initial conditions are attracted by the origin. Reverting back to the original system with time $\tau$, we conclude that for that system too the basin of attraction of the origin has full measure, provided $\beta<\alpha$ and $\gamma$ satisfies A.5. 


\section{B Action-angle variables}

In this section we detail the calculation of the action-angle variables for the simple pendulum in time $\tau$. More details on calculating action-angle variables can be found in [14, 32, 9. The simple pendulum has equation of motion given by

$$
\theta^{\prime \prime}+\alpha \sin \theta=0
$$

where the dashes represent derivative with respect to the scaled time $\tau$. The Hamiltonian for the simple pendulum in this notation is

$$
E=H\left(\theta, \theta^{\prime}\right)=\frac{1}{2}\left(\theta^{\prime}\right)^{2}-\alpha \cos \theta
$$

or, in terms of the usual notation for Hamiltonian dynamics,

$$
E=H(p, q)=\frac{1}{2} p^{2}-\alpha \cos q,
$$

where $q=\theta$ and $p=q^{\prime}=\theta^{\prime}$. Rearranging this for $p$ we obtain $p= \pm p(E, q)$, with

$$
p(E, q)=\sqrt{2(E+\alpha \cos q)}=\sqrt{2 \alpha\left(E_{0}+\cos q\right)},
$$

where $E_{0}=E / \alpha$. It is clear that there are two types of dynamics, oscillatory dynamics when $E_{0}<1$ and rotational dynamics when $E_{0}>1$, separated at a separatrix when $E_{0}=1$, for which no action-angle variables exist.

\section{B.1 Librations}

We first consider the case $E_{0}<1$. The action variable is

$$
I=\frac{1}{2 \pi} \oint p \mathrm{~d} q=\frac{2}{\pi} \sqrt{2 \alpha} \int_{0}^{q_{1}} \sqrt{E_{0}+\cos q} \mathrm{~d} q=\frac{8}{\pi} \sqrt{\alpha}\left[\left(k_{1}^{2}-1\right) \mathbf{K}\left(k_{1}\right)+\mathbf{E}\left(k_{1}\right)\right],
$$

where $k_{1}^{2}=\left(E_{0}+1\right) / 2$ and $q_{1}=\arccos \left(-E_{0}\right)$. The functions $\mathbf{K}(k)$ and $\mathbf{E}(k)$ are the complete elliptic integrals of the first and second kinds respectively.

The angle variable $\varphi$ can be found as follows

$$
\varphi^{\prime}=\frac{\partial H}{\partial I}=\frac{\mathrm{d} E}{\mathrm{~d} I}=\left(\frac{\mathrm{d} I}{\mathrm{~d} E}\right)^{-1},
$$

so that

Hence we have

$$
\frac{\mathrm{d} I}{\mathrm{~d} E}=\frac{\mathrm{d}}{\mathrm{d} E} \frac{2}{\pi} \int_{0}^{q_{1}} \sqrt{E+\alpha \cos q} \mathrm{~d} q=\frac{2}{\pi \sqrt{\alpha}} \mathbf{K}\left(k_{1}\right) .
$$

$$
\varphi(\tau)=\frac{\pi}{2 \mathbf{K}\left(k_{1}\right)} \sqrt{\alpha}\left(\tau-\tau_{0}\right) .
$$

Take $s=\sin (q / 2)$; then using equation $(\overline{B .2})$ it is easy to show that

$$
\left(s^{\prime}\right)^{2}=\frac{g}{l}\left(1-s^{2}\right)\left(k_{1}^{2}-s^{2}\right) .
$$

Integrating using the Jacobi elliptic functions

$$
s(\tau)=k_{1} \operatorname{sn}\left(\sqrt{\frac{g}{l}}\left(\tau-\tau_{0}\right), k_{1}\right),
$$

the expresssion can then be rearranged to achieve the following result:

$$
q=2 \arcsin \left[k_{1} \operatorname{sn}\left(\frac{2 \mathbf{K}\left(k_{1}\right)}{\pi} \varphi, k_{1}\right)\right], \quad p=2 k_{1} \sqrt{\alpha} \operatorname{cn}\left(\frac{2 \mathbf{K}\left(k_{1}\right)}{\pi} \varphi, k_{1}\right),
$$

which coincide with equations (2.4). By using (E.3) in Appendix E one obtains from B.5

$$
\frac{\partial I}{\partial k_{1}}=\frac{8}{\pi} k_{1} \mathbf{K}\left(k_{1}\right) \sqrt{\alpha}
$$

a relation which has been used to derive 2.12 . 


\section{B.2 Rotations}

In the case of rotational dynamics we have

$$
I=\frac{1}{2 \pi} \int_{0}^{2 \pi} p \mathrm{~d} q=\frac{1}{2 \pi} \sqrt{\alpha} \int_{0}^{2 \pi} \sqrt{E_{0}+\cos q} \mathrm{~d} q=\frac{4}{k_{2} \pi} \sqrt{\alpha} \mathbf{E}\left(k_{2}\right),
$$

where this time we let $k_{2}^{2}=2 /\left(E_{0}+1\right)=1 / k_{1}^{2}$. The angle variable $\varphi$ can similarly be found using (B.6), where $\mathrm{d} I / \mathrm{d} E$ can be similarly calculated as

$$
\frac{\mathrm{d} I}{\mathrm{~d} E}=\frac{\mathrm{d}}{\mathrm{d} E} \frac{1}{2 \pi} \int_{0}^{2 \pi} \sqrt{E+\alpha \cos q} \mathrm{~d} q=\frac{k_{2}}{\pi \sqrt{\alpha}} \mathbf{K}\left(k_{2}\right),
$$

which hence gives

$$
\varphi(\tau)=\frac{\pi}{\mathbf{K}\left(k_{2}\right)} \sqrt{\alpha} \frac{\left(\tau-\tau_{0}\right)}{k_{2}} .
$$

Using $B .9$ and the definition of $k_{2}$, for the rotating solutions we find that

$$
s(\tau)=\operatorname{sn}\left(\sqrt{\alpha} \frac{\left(\tau-\tau_{0}\right)}{k_{2}}, k_{2}\right),
$$

and similarly, by simple rearrangement, we find that

$$
q=2 \arcsin \left[\operatorname{sn}\left(\frac{\mathbf{K}\left(k_{2}\right)}{\pi} \varphi, k_{2}\right)\right], \quad p=\frac{2}{k_{2}} \sqrt{\alpha} \operatorname{dn}\left(\frac{\mathbf{K}\left(k_{2}\right)}{\pi} \varphi, k_{2}\right),
$$

which again yields equations 2.6 By using E.3 in Appendix E, one obtains from B.13

$$
\frac{\partial I}{\partial k_{2}}=-\frac{4}{\pi k_{2}^{2}} \mathbf{K}\left(k_{2}\right) \sqrt{\alpha} .
$$

which has been used to derive 2.23 .

\section{Jacobian determinant}

Here we compute the entries of the Jacobian matrix $J$ of the transformation to action-angle variables, which will be used in the next Appendix. As a by-product we check that $J$ determinant equal to 1 , that is

$$
\frac{\partial q}{\partial \varphi} \frac{\partial p}{\partial I}-\frac{\partial q}{\partial I} \frac{\partial p}{\partial \varphi}=1
$$

For further details on the proof of (C.1) we refer the reader to [9], where the calculations are given in great detail. The derivative with respect to $\varphi$ is straightforward in both the libration and rotation case, however the dependence of $p$ and $q$ on the action $I$ is less obvious. That said, the dependence of $p$ and $q$ on $k_{1}$ in the oscillating case and $k_{2}$ in the rotating case is clear and we know the relationship between $I$ and $k$ in both cases, hence the derivative of the Jacobi elliptic functions can be calculated by using that

$$
\frac{\partial}{\partial I}=\frac{\partial k}{\partial I} \frac{\partial}{\partial k}+\frac{\partial u}{\partial I} \frac{\partial}{\partial u}=\frac{\partial k}{\partial I}\left(\frac{\partial}{\partial k}+\frac{\partial u}{\partial k} \frac{\partial}{\partial u}\right),
$$

where $u$ is the first argument of the functions, i.e $\operatorname{sn}(u, k)$, etc. Then for the oscillations we have

$$
\begin{aligned}
\frac{\partial q}{\partial I} & =\frac{\pi}{4 k_{1} \mathbf{K}\left(k_{1}\right) \sqrt{\alpha}}\left[\frac{\operatorname{sn}(\cdot)}{\operatorname{dn}(\cdot)}+\frac{2 \mathbf{E}\left(k_{1}\right) \varphi \operatorname{cn}(\cdot)}{\pi k_{1}^{\prime 2}}+\frac{k_{1}^{2} \operatorname{sn}(\cdot) \operatorname{cn}^{2}(\cdot)}{k_{1}^{\prime 2} \operatorname{dn}(\cdot)}-\frac{\mathbf{E}(\cdot) \operatorname{cn}(\cdot)}{k_{1}^{\prime 2}}\right] \\
\frac{\partial p}{\partial I} & =\frac{\pi}{4 k_{1} \mathbf{K}\left(k_{1}\right)}\left[\operatorname{cn}(\cdot)-\frac{2 \mathbf{E}\left(k_{1}\right) \varphi \operatorname{sn}(\cdot) \operatorname{dn}(\cdot)}{\pi k_{1}^{\prime 2}}-\frac{k_{1}^{2} \operatorname{sn}^{2}(\cdot) \operatorname{cn}(\cdot)}{k_{1}^{\prime 2}}+\frac{\mathbf{E}(\cdot) \operatorname{sn}(\cdot) \operatorname{dn}(\cdot)}{k_{1}^{\prime 2}}\right], \\
\frac{\partial q}{\partial \varphi} & =\frac{4 k_{1} \mathbf{K}\left(k_{1}\right) \operatorname{cn}(\cdot)}{\pi} \\
\frac{\partial p}{\partial \varphi} & =-\sqrt{\alpha} \frac{4 k_{1} \mathbf{K}\left(k_{1}\right) \operatorname{sn}(\cdot) \operatorname{dn}(\cdot)}{\pi}
\end{aligned}
$$


where $(\cdot)=\left(\frac{2 \mathbf{K}\left(k_{1}\right) \varphi}{\pi}, k_{1}\right)$ and $k_{1}^{\prime}=\sqrt{1-k_{1}^{2}}$. From the above it is easy to check that equation (C.1) is satisfied. Similarly, for the rotations we have

$$
\begin{aligned}
\frac{\partial q}{\partial I} & =-\frac{\pi k_{2}^{2}}{2 \mathbf{K}\left(k_{2}\right) \sqrt{\alpha}}\left[\frac{\varphi \mathbf{E}\left(k_{2}\right) \operatorname{dn}(\cdot)}{\pi k_{2} k_{2}^{\prime 2}}+\frac{k_{2} \operatorname{sn}(\cdot) \operatorname{cn}(\cdot)}{k_{2}^{\prime 2}}-\frac{\mathbf{E}(\cdot) \operatorname{dn}(\cdot)}{k_{2} k_{2}^{\prime 2}}\right], \\
\frac{\partial p}{\partial I} & =\frac{\pi k_{2}^{2}}{2 \mathbf{K}\left(k_{2}\right)}\left[\frac{\operatorname{dn}(\cdot)}{k_{2}^{2}}+\frac{\varphi \mathbf{E}\left(k_{2}\right) \operatorname{sn}(\cdot) \operatorname{cn}(\cdot)}{\pi k_{2}^{\prime 2}}+\frac{\operatorname{sn}^{2}(\cdot) \operatorname{dn}(\cdot)}{k_{2}^{\prime 2}}-\frac{\mathbf{E}(\cdot) \operatorname{sn}(\cdot) \operatorname{cn}(\cdot)}{k_{2}^{\prime 2}}\right], \\
\frac{\partial q}{\partial \varphi} & =\frac{2 \mathbf{K}\left(k_{2}\right) \operatorname{dn}(\cdot)}{\pi} \\
\frac{\partial p}{\partial \varphi} & =-\sqrt{\alpha} \frac{2 k_{2} \mathbf{K}\left(k_{2}\right) \operatorname{sn}(\cdot) \operatorname{cn}(\cdot)}{\pi}
\end{aligned}
$$

where $(\cdot)=\left(\frac{\mathbf{K}\left(k_{2}\right)}{\pi} \varphi, k_{2}\right)$ and $k_{2}^{\prime}=\sqrt{1-k_{2}^{2}}$. It is once again easily checked from the above that C.1 is satisfied.

\section{Equations of motion for the perturbed system}

By (C.1) one has

$$
\left(\begin{array}{ll}
\partial \varphi / \partial q & \partial \varphi / \partial p \\
\partial I / \partial q & \partial I / \partial p
\end{array}\right)=\left(\begin{array}{cc}
\partial p / \partial I & -\partial q / \partial I \\
-\partial p / \partial \varphi & \partial q / \partial \varphi
\end{array}\right)
$$

We rewrite the equation 1.3 in the action-angle coordinates introduced in Appendix $B$ as follows.

\section{D.1 Librations}

In this section we want to write 1.3 in terms of the action-angle introduced in Appendix B By taking into account the forcing term $-\beta \cos \tau \cos \theta$ in 1.3 one finds

$$
\begin{aligned}
I^{\prime} & =\frac{\partial I}{\partial q} q^{\prime}+\frac{\partial I}{\partial p} p^{\prime}=-\frac{\partial p}{\partial \varphi} q^{\prime}+\frac{\partial q}{\partial \varphi} p^{\prime} \\
= & \frac{8 \beta k_{1}^{2} \mathbf{K}\left(k_{1}\right)}{\pi} \cos \left(\tau-\tau_{0}\right) \operatorname{sn}(\cdot) \operatorname{cn}(\cdot) \operatorname{dn}(\cdot), \\
\varphi^{\prime} & =\frac{\partial \varphi}{\partial q} q^{\prime}+\frac{\partial \varphi}{\partial p} p^{\prime}=\frac{\partial p}{\partial I} q^{\prime}-\frac{\partial q}{\partial I} p^{\prime} \\
= & \frac{\pi \sqrt{\alpha}}{2 \mathbf{K}\left(k_{1}\right)}-\frac{\pi \beta}{2 \mathbf{K}\left(k_{1}\right) \sqrt{\alpha}}\left[\operatorname{sn}^{2}(\cdot)+\frac{2 \mathbf{E}\left(k_{1}\right) \varphi \operatorname{sn}(\cdot) \operatorname{cn}(\cdot) \operatorname{dn}(\cdot)}{\pi k_{1}^{\prime 2}}\right. \\
& \left.\quad+\frac{k_{1}^{2} \operatorname{sn}^{2}(\cdot) \operatorname{cn}^{2}(\cdot)}{1-k_{1}^{2}}-\frac{\mathbf{E}(\cdot) \operatorname{sn}(\cdot) \operatorname{cn}(\cdot) \operatorname{dn}(\cdot)}{1-k_{1}^{2}}\right] \cos \left(\tau-\tau_{0}\right),
\end{aligned}
$$

where we have used the properties of the Jacobi elliptic functions in Appendix E As in Appendix C we are shortening $(\cdot)=\left(\frac{2 \mathbf{K}\left(k_{1}\right) \varphi}{\pi}, k_{1}\right)$.

We then wish to add the dissipative term $\gamma \theta^{\prime}$. This results in the following equations:

$$
\begin{aligned}
I^{\prime}=\frac{8 \beta k_{1}^{2} \mathbf{K}\left(k_{1}\right)}{\pi} \cos \left(\tau-\tau_{0}\right) \operatorname{sn}(\cdot) \operatorname{cn}(\cdot) \operatorname{dn}(\cdot)-\frac{8 \gamma k_{1}^{2} \sqrt{\alpha} \mathbf{K}\left(k_{1}\right)}{\pi} \operatorname{cn}^{2}(\cdot), \\
\begin{aligned}
\varphi^{\prime}=\frac{\pi \sqrt{\alpha}}{2 \mathbf{K}\left(k_{1}\right)} & -\frac{\pi \beta}{2 \mathbf{K}\left(k_{1}\right) \sqrt{\alpha}}\left[\operatorname{sn}^{2}(\cdot)+\frac{2 \mathbf{E}\left(k_{1}\right) \varphi \operatorname{sn}(\cdot) \operatorname{cn}(\cdot) \operatorname{dn}(\cdot)}{\pi\left(1-k_{1}^{2}\right)}\right. \\
& \left.+\frac{k_{1}^{2} \operatorname{sn}^{2}(\cdot) \operatorname{cn}^{2}(\cdot)}{1-k_{1}^{2}}-\frac{\mathbf{E}(\cdot) \operatorname{sn}(\cdot) \operatorname{cn}(\cdot) \operatorname{dn}(\cdot)}{1-k_{1}^{2}}\right] \cos \left(\tau-\tau_{0}\right) \\
& +\frac{\gamma \pi \operatorname{cn}(\cdot)}{2 \mathbf{K}\left(k_{1}\right)}\left[\frac{\operatorname{sn}(\cdot)}{\operatorname{dn}(\cdot)}+\frac{2 \mathbf{E}\left(k_{1}\right) \varphi \operatorname{cn}(\cdot)}{\pi\left(1-k_{1}^{2}\right)}+\frac{k_{1}^{2} \operatorname{sn}(\cdot) \operatorname{cn}^{2}(\cdot)}{\left(1-k_{1}^{2}\right) \operatorname{dn}(\cdot)}-\frac{\mathbf{E}(\cdot) \operatorname{cn}(\cdot)}{1-k_{1}^{2}}\right] .
\end{aligned}
\end{aligned}
$$

Using the property that, see [26, $\mathbf{E}(u, k)=\mathbf{E}(k) u / \mathbf{K}(k)+\mathbf{Z}(u, k)$ we arrive at equations 2.9$)$. The function $\mathbf{Z}(u, k)$ is the Jacobi zeta function, which is periodic with period $2 \mathbf{K}(k)$ in $u$. 


\section{D.2 Rotations}

The presence of the forcing term leads to te equations

$$
\begin{aligned}
I^{\prime}=-\frac{\partial p}{\partial \varphi} q^{\prime} & +\frac{\partial q}{\partial \varphi} p^{\prime}=\frac{4 \beta \mathbf{K}\left(k_{2}\right)}{\pi} \cos \left(\tau-\tau_{0}\right) \operatorname{sn}(\cdot) \operatorname{cn}(\cdot) \operatorname{dn}(\cdot) \\
\varphi^{\prime}=\frac{\partial p}{\partial I} \dot{q} & -\frac{\partial q}{\partial I} \dot{p}=\frac{\pi \sqrt{\alpha}}{k_{2} \mathbf{K}\left(k_{2}\right)}+\frac{\pi k_{2} \beta}{\sqrt{\alpha} \mathbf{K}\left(k_{2}\right)}\left[\frac{\mathbf{E}\left(k_{2}\right) \varphi \operatorname{sn}(\cdot) \operatorname{cn}(\cdot) \operatorname{dn}(\cdot)}{\pi\left(1-k_{2}^{2}\right)}\right. \\
& \left.+\frac{k_{2}^{2} \operatorname{sn}^{2}(\cdot) \mathrm{cn}^{2}(\cdot)}{1-k_{2}^{2}}-\frac{\mathbf{E}(\cdot) \operatorname{sn}(\cdot) \operatorname{cn}(\cdot) \operatorname{dn}(\cdot)}{1-k_{2}^{2}}\right] \cos \left(\tau-\tau_{0}\right) .
\end{aligned}
$$

Again, if we wish to add a dissipative term, we arrive at the equations

$$
\begin{aligned}
I^{\prime}=\frac{4 \beta \mathbf{K}\left(k_{2}\right)}{\pi} & \cos \left(\tau-\tau_{0}\right) \operatorname{sn}(\cdot) \operatorname{cn}(\cdot) \operatorname{dn}(\cdot)-\frac{4 \gamma \sqrt{\alpha} \mathbf{K}\left(k_{2}\right)}{\pi k_{2}} \operatorname{dn}^{2}(\cdot), \\
\varphi^{\prime}=\frac{\pi \sqrt{\alpha}}{k_{2} \mathbf{K}\left(k_{2}\right)} & +\frac{\pi k_{2} \beta}{\sqrt{\alpha} \mathbf{K}\left(k_{2}\right)}\left[\frac{\mathbf{E}\left(k_{2}\right) \varphi \operatorname{sn}(\cdot) \operatorname{cn}(\cdot) \operatorname{dn}(\cdot)}{\pi\left(1-k_{2}^{2}\right)}\right. \\
& \left.+\frac{k_{2}^{2} \operatorname{sn}^{2}(\cdot) \operatorname{cn}^{2}(\cdot)}{1-k_{2}^{2}}-\frac{\mathbf{E}(\cdot) \operatorname{sn}(\cdot) \operatorname{cn}(\cdot) \operatorname{dn}(\cdot)}{1-k_{2}^{2}}\right] \cos \left(\tau-\tau_{0}\right) \\
& -\frac{\gamma \pi}{\mathbf{K}\left(k_{2}\right)}\left[\frac{\mathbf{E}\left(k_{2}\right) \varphi \operatorname{dn}^{2}(\cdot)}{\pi\left(1-k_{2}^{2}\right)}+\frac{k_{2}^{2} \operatorname{sn}(\cdot) \operatorname{cn}(\cdot) \operatorname{dn}(\cdot)}{1-k_{2}^{2}}-\frac{\mathbf{E}(\cdot) \mathrm{dn}^{2}(\cdot)}{1-k_{2}^{2}}\right] .
\end{aligned}
$$

Again using that $\mathbf{E}(u, k)=\mathbf{E}(k) u / \mathbf{K}(k)+\mathbf{Z}(u, k)$ we arrive at the equations 2.22).

\section{E Useful properties of the elliptic functions}

The complete integrals of the first and second kind are, respectively,

$$
\mathbf{K}(k)=\int_{0}^{\pi / 2} \frac{\mathrm{d} \psi}{\sqrt{1-k^{2} \sin ^{2} \psi}}, \quad \mathbf{E}(k)=\int_{0}^{\pi / 2} \mathrm{~d} \psi \sqrt{1-k^{2} \sin ^{2} \psi},
$$

whereas the incomplete elliptic integral of the second kind is

$$
\mathbf{E}(u, k)=\int_{0}^{\operatorname{sn}(u, k)} \mathrm{d} x \frac{\sqrt{1-k^{2} x^{2}}}{\sqrt{1-x^{2}}} .
$$

One has

$$
\frac{\partial \mathbf{K}(k)}{\partial k}=\frac{1}{k}\left(\frac{\mathbf{E}(k)}{1-k^{2}}-\mathbf{K}(k)\right), \quad \frac{\partial \mathbf{E}(k)}{\partial k}=\frac{1}{k}(\mathbf{E}(k)-\mathbf{K}(k)) .
$$

The following properties of the Jacobi elliptic functions have been used in the previous sections. The derivatives with respect to the first arguments are

$$
\begin{aligned}
& \frac{\partial}{\partial u} \operatorname{sn}(u, k)=\operatorname{cn}(u, k) \operatorname{dn}(u, k), \\
& \frac{\partial}{\partial u} \operatorname{cn}(u, k)=-\operatorname{sn}(u, k) \operatorname{dn}(u, k), \\
& \frac{\partial}{\partial u} \operatorname{dn}(u, k)=-k^{2} \operatorname{sn}(u, k) \operatorname{cn}(u, k),
\end{aligned}
$$

while the derivatives with respect to the elliptic modulus are

$$
\begin{aligned}
\frac{\partial}{\partial k} \operatorname{sn}(u, k) & =\frac{u}{k} \operatorname{cn}(u, k) \operatorname{dn}(u, k)+\frac{k}{k^{\prime 2}} \operatorname{sn}(u, k) \operatorname{cn}^{2}(u, k)-\frac{1}{k k^{\prime 2}} \mathbf{E}(u, k) \operatorname{cn}(u, k) \operatorname{dn}(u, k), \\
\frac{\partial}{\partial k} \operatorname{cn}(u, k) & =-\frac{u}{k} \operatorname{sn}(u, k) \operatorname{dn}(u, k)-\frac{k}{k^{\prime 2}} \operatorname{sn}^{2}(u, k) \operatorname{cn}(u, k)+\frac{1}{k^{\prime 2}} \mathbf{E}(u, k) \operatorname{sn}(u, k) \operatorname{dn}(u, k), \\
\frac{\partial}{\partial k} \operatorname{dn}(u, k) & =-k u \operatorname{sn}(u, k) \operatorname{cn}(u, k)-\frac{k}{k^{\prime 2}} \operatorname{sn}^{2}(u, k) \operatorname{dn}(u, k)+\frac{k}{k^{\prime 2}} \mathbf{E}(u, k) \operatorname{sn}(u, k) \operatorname{cn}(u, k),
\end{aligned}
$$


where $k^{\prime 2}=1-k^{2}$.

Finding the value of $\Delta$ for rotations in Section 2 requires use of

$$
\int_{0}^{x_{1}} \operatorname{dn}^{2}(x, k) \mathrm{d} x=\int_{0}^{\operatorname{sn}\left(x_{1}, k\right)} \frac{\sqrt{1-k^{2} \hat{x}^{2}}}{\sqrt{1-\hat{x}^{2}}} \mathrm{~d} \hat{x}=\mathbf{E}\left(x_{1}, k\right) .
$$

In the case of librations we also require the relation $k^{2} \mathrm{cn}^{2}(\cdot)+\left(1-k^{2}\right)=\mathrm{dn}^{2}(\cdot)$.

The integral for $\Gamma_{1}\left(\tau_{0} ; p, q\right)$ in equation 2.20 is found by

$$
\begin{aligned}
\Gamma_{1}\left(\tau_{0} ; p, q\right)= & \frac{1}{T} \int_{0}^{T} \operatorname{sn}(\sqrt{\alpha} \tau) \operatorname{cn}(\sqrt{\alpha} \tau) \operatorname{dn}(\sqrt{\alpha} \tau) \cos \left(\tau-\tau_{0}\right) \mathrm{d} \tau \\
= & \frac{\cos \left(\tau_{0}\right)}{T} \int_{0}^{T} \operatorname{sn}(\sqrt{\alpha} \tau) \operatorname{cn}(\sqrt{\alpha} \tau) \operatorname{dn}(\sqrt{\alpha} \tau) \cos (\tau) \mathrm{d} \tau \\
& +\frac{\sin \left(\tau_{0}\right)}{T} \int_{0}^{T} \operatorname{sn}(\sqrt{\alpha} \tau) \operatorname{cn}(\sqrt{\alpha} \tau) \operatorname{dn}(\sqrt{\alpha} \tau) \sin (\tau) \mathrm{d} \tau \\
= & \frac{\sin \left(\tau_{0}\right)}{T} \int_{0}^{T} \operatorname{sn}(\sqrt{\alpha} \tau) \operatorname{cn}(\sqrt{\alpha} \tau) \operatorname{dn}(\sqrt{\alpha} \tau) \sin (\tau) \mathrm{d} \tau,
\end{aligned}
$$

where $T=2 \pi q=4 \mathbf{K}\left(k_{1}\right) p$.

The Jacobi elliptic functions can be expanded in a Fourier series as

$$
\begin{array}{r}
\operatorname{sn}(u, k)=\frac{2 \pi}{k \mathbf{K}(k)} \sum_{n=1}^{\infty} \frac{\mathfrak{q}^{n-1 / 2}}{1-\mathfrak{q}^{2 n-1}} \sin \left(\frac{(2 n-1) \pi u}{2 \mathbf{K}(k)}\right), \\
\operatorname{cn}(u, k)=\frac{2 \pi}{k \mathbf{K}(k)} \sum_{n=1}^{\infty} \frac{\mathfrak{q}^{n-1 / 2}}{1+\mathfrak{q}^{2 n-1}} \cos \left(\frac{(2 n-1) \pi u}{2 \mathbf{K}(k)}\right), \\
\operatorname{dn}(u, k)=\frac{\pi}{2 \mathbf{K}(k)}+\frac{2 \pi}{\mathbf{K}(k)} \sum_{n=1}^{\infty} \frac{\mathfrak{q}^{n}}{1-\mathfrak{q}^{2 n}} \cos \left(\frac{2 n \pi u}{2 \mathbf{K}(k)}\right),
\end{array}
$$

where $\mathfrak{q}$ is the nome, defined as

$$
\mathfrak{q}=\exp \left(-\frac{\pi \mathbf{K}\left(k^{\prime}\right)}{\mathbf{K}(k)}\right)
$$

with $k^{\prime}=\sqrt{1-k^{2}}$.

In the calculation of $\left\langle R^{(n)}\right\rangle$ for $n \geq 2$, when the pendulum is in libration, we require the evaluation of the integrals

$$
\frac{1}{T} \int_{0}^{T} \frac{2 \mathbf{K}\left(k_{1}\right)}{\sqrt{\alpha} \pi} \frac{\partial}{\partial \tau}\left(\operatorname{cn}^{2}(\sqrt{\alpha} \tau)\right) \mathrm{d} \tau=0
$$

and

$$
\begin{aligned}
\frac{1}{T} \int_{0}^{T} & \frac{2 \mathbf{K}\left(k_{1}\right)}{\sqrt{\alpha} \pi} \frac{\partial}{\partial \tau}(\operatorname{sn}(\sqrt{\alpha} \tau) \operatorname{cn}(\sqrt{\alpha} \tau) \operatorname{dn}(\sqrt{\alpha} \tau)) \cos \left(\tau-\tau_{0}\right) \mathrm{d} \tau \\
= & \frac{1}{T} \int_{0}^{T} \frac{2 \mathbf{K}\left(k_{1}\right)}{\sqrt{\alpha} \pi} \operatorname{sn}(\sqrt{\alpha} \tau) \operatorname{cn}(\sqrt{\alpha} \tau) \operatorname{dn}(\sqrt{\alpha} \tau) \sin \left(\tau-\tau_{0}\right) \mathrm{d} \tau \\
= & \frac{\cos \left(\tau_{0}\right)}{T} \int_{0}^{T} \frac{2 \mathbf{K}\left(k_{1}\right)}{\sqrt{\alpha} \pi} \operatorname{sn}(\sqrt{\alpha} \tau) \operatorname{cn}(\sqrt{\alpha} \tau) \operatorname{dn}(\sqrt{\alpha} \tau) \sin (\tau) \mathrm{d} \tau \\
& \quad-\frac{\sin \left(\tau_{0}\right)}{T} \int_{0}^{T} \frac{2 \mathbf{K}\left(k_{1}\right)}{\sqrt{\alpha} \pi} \operatorname{sn}(\sqrt{\alpha} \tau) \operatorname{cn}(\sqrt{\alpha} \tau) \operatorname{dn}(\sqrt{\alpha} \tau) \cos (\tau) \mathrm{d} \tau
\end{aligned}
$$

where $T=2 \pi q=4 \mathbf{K}\left(k_{1}\right) p$. The integral multiplying $\sin \left(\tau_{0}\right)$ vanishes due to parity and hence

$$
\frac{2 \mathbf{K}\left(k_{1}\right)}{\sqrt{\alpha} \pi T} \int_{0}^{T} \frac{\partial}{\partial \tau}(\operatorname{sn}(\sqrt{\alpha} \tau) \operatorname{cn}(\sqrt{\alpha} \tau) \operatorname{dn}(\sqrt{\alpha} \tau)) \cos \left(\tau-\tau_{0}\right) \mathrm{d} \tau=\frac{2 \mathbf{K}\left(k_{1}\right)}{\sqrt{\alpha} \pi} \cos \left(\tau_{0}\right) G_{1}(p, q) .
$$




\section{References}

[1] M.V. Bartuccelli, A. Berretti, J.H.B. Deane, G. Gentile, S.A. Gourley, Selection rules for periodic orbits and scaling laws for a driven damped quartic oscillator, Nonlinear Anal. Real World Appl. 9 (2008), no. 5, 1966-1988.

[2] M.V. Bartuccelli, J.H.B. Deane, G. Gentile, Globally and locally attractive solutions for quasiperiodically forced systems, J. Math. Anal. Appl. 328 (2007), no. 1, 699-714.

[3] M.V. Bartuccelli, J.H.B. Deane, G. Gentile, Attractiveness of periodic orbits in parametrically forced systems with time-increasing friction, J. Math. Phys. 53 (2012), no.10, 102703, 27 pp.

[4] M.V. Bartuccelli, J.H.B. Deane, G. Gentile, S.A. Gourley, Global attraction to the origin in a parametrically-driven nonlinear oscillator, Appl. Math. Comput. 153 (2004), no. 1, 1-11.

[5] M.V. Bartuccelli, G. Gentile, K.V. Georgiou, On the dynamics of a vertically driven damped planar pendulum, Proc. R. Soc. Lond. A 457 (2001), no. 2016, 3007-3022.

[6] M.V. Bartuccelli, G. Gentile, K.V. Georgiou, On the stability of the upside-down pendulum with damping, Proc. R. Soc. Lond. A 458 (2002), no. 2018, 255-269.

[7] M.V. Bartuccelli, G. Gentile, K.V. Georgiou, KAM theory, Lindstedt series and the stability of the upside-down pendulum, Discrete Contin. Dyn. Syst. 9 (2003), no. 2, 413-426.

[8] F. Bowman, Introduction to elliptic functions with applications, English Universities Press, London, 1953.

[9] A.J. Brizard, Jacobi zeta function and action-angle coordinates for the pendulum, Commun. Nonlinear Sci. Numer. Simul. 18 (2013), 511-518.

[10] K. Brown, Topics in calculus and probability, Self-published on Lulu (2012), 64-77. Also available at: www.mathpages.com.

[11] P.F. Byrd, M.D. Friedman, Handbook of elliptic integrals for engineers and scientists, Second edition revised, Springer, New York-Heidelberg, 1971.

[12] J.R. Cannon, K. Miller, Some problems in numerical analytic continuation, J. Soc. Indust. Appl. Math. Ser. B Numer. Anal. 2 (1965), no. 1, 87-98.

[13] Sh.N. Chow, J.K. Hale, Methods of bifurcation theory, Grundlehren der Mathematischen Wissenschaften 251, Springer, New York-Berlin, 1982.

[14] A. Fasano, S. Marmi, Analytical mechanics, Oxford University Press, Oxford, 2006.

[15] U. Feudel, C. Grebogi, Why are chaotic attractors rare in multistable systems, Phys. Rev. E 91 (2003), 134102, 4pp.

[16] U. Feudel, C. Grebogi, B.R. Hunt, J.A. Yorke, Map with more than 100 coexisting low-period periodic attractors, Phys. Rev. E 54 (1996), 71-81.

[17] E. Gallicchio, S.A. Egorov, B.J. Berne, On the application of numerical analytic continuation methods to the study of quantum mechanical vibrational relaxation processes, J. Chem. Phys. 109 (1998), no. $18,7745-7755$.

[18] G. Gentile, M. Bartuccelli, J. Deane, Bifurcation curves of subharmonic solutions and Melnikov theory under degeneracies, Rev. Math. Phys. 19 (2007), no. 3, 307-348.

[19] G. Gentile, G. Gallavotti, Degenerate elliptic resonances, Comm. Math. Phys. 257 (2005), no. 2, 319362

[20] M. Ghil, G. Wolansky, Non-Hamiltonian perturbations of integrable systems and resonance trapping, SIAM J. Appl. Math. 52 (1992), no. 4, 1148-1171.

[21] J. Guckenheimer, Ph. Holmes, Nonlinear oscillations, dynamical systems, and bifurcations of vector fields, Revised and corrected reprint of the 1983 original, Applied Mathematical Sciences 42, Springer, New York, 1990. 
[22] D.W. Jordan, P. Smith, Nonlinear ordinary differential equations. An introduction for scientists and engineers, Fourth edition, Oxford University Press, Oxford, 2007.

[23] K. Kodaira, Complex analysis, Cambridge University Press, Cambridge, 2007.

[24] N.N. Krasovsky, Problems of the Theory of Stability of Motion, Stanford University Press, Stanford, 1963.

[25] L. D. Landau, E. M. Lifshitz, Mechanics, Third Editon, Pergamon Press, Oxford, 1976.

[26] D.F. Lawden, Elliptic functions and applications, Springer, New York, 1989.

[27] W. Magnus, S. Winkler, Hill's equation, Second edition - reprint, Dover Publications, New York, 2004.

[28] C. D. Murray, S. F. Dermott, Solar System Dynamics, Cambridge University Press, Cambridge, 2001.

[29] R.H. Myers, S.L. Myers, R.E. Walpole, Probability and statistics for engineers and scientists, Sixth edition, Prentice Hall, London, 1998.

[30] R.K. Nagle, E.B. Saff, A.D. Snider, Fundamentals of differential equations, Eight edition, AddisonWesley, Boston, 2012.

[31] J. Palis, A global view of dynamics and a conjecture on the denseness of finitude of attractors, Astérisque 261, (2000), 339-351.

[32] I. Percival, D. Richards, Introduction to dynamics, First edition - reprint, Cambridge University Press, Cambridge, 1994.

[33] Ch.S. Rodrigues, A.P.S. de Moura, C. Grebogi, Emerging attractors and the transition from dissipative to conservative dynamics, Phys. Rev. E 80 (2009), no. 2, 026205, 4pp.

[34] I. Stewart, D. Tall, Complex analysis, Cambridge University Press, Cambridge, 1983.

[35] G.N. Watson, E. T. Whittaker, A course of modern analysis, Fourth edition - reprint, Cambridge University Press, Cambridge, 1947.

[36] J.A. Wright, J.H.B. Deane, M. Bartuccelli, G. Gentile, Analytic continuation applied to the problem of the pendulum with vertically oscillating support, in preparation. 